\title{
The AGB phase-transition outside the local group: $K$-band observations of young star clusters in NGC 7252
}

\author{
C. Maraston ${ }^{1}$, M. Kissler-Patig ${ }^{2}$, J. P. Brodie ${ }^{3}$, P. Barmby ${ }^{4}$, and J. P. Huchra ${ }^{4}$ \\ 1 Universitäts-Sternwarte der Ludwig-Maximilians-Universität, Scheinerstr. 1, 81679 München, Germany \\ 2 European Southern Observatory, Karl-Schwarzschild-Str. 2, 85748 Garching, Germany \\ e-mail: mkissler@eso.org \\ 3 University of California Observatories / Lick Observatory, University of California, Santa Cruz, CA 95064, USA \\ e-mail: brodie@ucolick.org \\ 4 Havard-Smithsonian Center for Astrophysics, 60 Garden St., Cambridge, MA 02138, USA \\ e-mail: pbarmby@cfa.harvard.edu; huchra@cfa.harvard.edu
}

Received 5 September 2000 / Accepted 26 January 2001

\begin{abstract}
We have extended the study of the young star clusters observed in the merger remnant galaxy NGC 7252 by obtaining $K$ band photometry for these clusters. Our $K$ band data significantly complement the optical photometry and spectroscopy in the literature: $K$ band data are fundamental to study the Asymptotic Giant Branch (AGB) population of these clusters, since the AGB phase transition (occuring between the age of $\sim 200$ Myr and $\sim 1$ Gyr) causes abrupt changes in the near-infrared luminosity of the clusters while producing only small changes in the optical. Therefore, the e.g. $V-K$ colour is ideal to study this evolutionary phase of stellar populations. For the present analysis we present models for Simple Stellar Populations which include the contribution of the AGB stellar phase, calibrated with the young and intermediate age star clusters of the Magellanic Clouds. The comparison with the colour distribution of the NGC 7252 star clusters shows that they are indeed intermediate age clusters undergoing the AGB phase transition. The AGB phase transition is observed for the first time outside the Local Group. Most of the studied clusters span the very narrow age range 300-500 Myr, and likely have metallicities $0.5-1 Z_{\odot}$. A very important exception is the cluster W32, which has already completed its AGB epoch, its colours being consistent with an age of $\sim 1-2$ Gyr. This impacts on the duration of the merger-induced starburst. The strengths of the magnesium and iron lines in the spectrum of the best observed cluster W3, and in the spectrum of the diffuse central light of NGC 7252, do not show an overabundance in $\alpha$-elements, in contrast to the bulk stellar population of elliptical galaxies.
\end{abstract}

Key words. galaxies: star clusters: stellar content: interactions - stars: AGB and post-AGB - stars: evolution

\section{Introduction}

Over the last decade, many systems of luminous star clusters have been discovered in merging and merger remnant galaxies (Lutz 1991; Holtzman et al. 1992; see also Schweizer 1998 for a review). Many of these appear to be globular clusters based on their compactness (small effective radius). They are thought to be young systems $(t<1 \mathrm{Gyr})$ on the basis of their blue broadband colours and high luminosities. The low ages support the view that they formed during the past merger. The typical duration of the merger-induced star formation episode is about a few hundred million years (Mihos \& Hernquist 1996). These newly formed globular clusters provide the

Send offprint requests to: C. Maraston,

e-mail: maraston@usm.uni-muenchen.de opportunity to study the process of globular cluster formation, and the early phases of the evolution of their stellar populations.

From the theoretical side, a stellar population aging to $t \sim 200-400 \mathrm{Myr}$ (intermediate-age range) abruptly develops a well populated Asymptotic Giant Branch (AGB), and consequently displays very red colours. When the age exceeds $\sim 1$ Gyr, the contribution of AGB stars sharply decreases. This very short epoch is referred to as the AGB phase-transition (Renzini 1981). The observational counterpart of this theoretical prediction is represented by the intermediate-age GCs in the Magellanic Clouds, which span a large range in $V-K$ (from $\sim 1$ to $\sim 3 \mathrm{mag}$ ), as a consequence of the AGB phase-transition (see e.g. Persson et al. 1983; Frogel et al. 1990). The Magellanic Clouds are the only sites in which the AGB phase transition has been 
observed so far. Recent mergers are the natural places to further investigate the occurrence of such a phenomenon.

The galaxy NGC 7252 is a prototypical remnant of two merged disk-like galaxies (Fritze-v Alvensleben \& Gerhard 1994a,b; Schweizer 1998), containing very bright young globular clusters (Schweizer 1982; Whitmore et al. 1993). A deep optical photometric study with $H S T$ was carried out by Miller et al. (1997), who conveniently divided the star clusters of NGC 7252 into two populations. The "outer" population contains objects with projected galactocentric distances between $6^{\prime \prime}$ and $\sim 120^{\prime \prime}$, the "inner" population contains objects within $6^{\prime \prime}$ of the nucleus. By analyzing the object colours and using a reddening-free parameter, Miller et al. (1997) conclude that the inner sample mainly consists of very young OB associations, while the outer sample is composed by bright blue (young) globular clusters and by fainter reddish (old) globular clusters. Schweizer \& Seitzer (1998) performed a spectroscopic study of a sub-sample of globular clusters belonging to the outer blue population. Ages for these clusters were determined via comparison of the strong observed Balmer lines with various sets of models, and found to be in the narrow range 400-600 Myr. The ages determined from $V, I$ optical photometry were found to be $20 \%$ smaller than the spectroscopic ones.

If the outer blue population of NGC 7252 contains intermediate-age clusters, the evidence for AGB phase transition is expected among these clusters. With this in mind, we obtained $K$ magnitudes for a sample of globular clusters belonging to the outer blue sample. The $K$ photometry, not included in previous studies, was motivated by the fact that AGB stars mainly emit in the infrared (see e.g. Persson et al. 1983). Complementing with published optical photometry, we analyze the data in the two-colour diagram $V-K$ vs. $B-V$, using Simple Stellar Population models calibrated with the intermediate-age star clusters in Magellanic Clouds (Maraston 1998). The spectroscopy by Schweizer \& Seitzer (1998) is used here to further constrain the cluster metallicities, and to investigate the element abundances. The galaxy NGC 7252 is the only remnant so far to have been modelled in detail using $N$-body simulations (Fritze-v Alvensleben \& Gerhard 1994b; Hibbard \& Mihos 1995). The derived age distribution of our cluster sample can therefore be compared with the predictions of these simulations.

The paper is organized as follows. Section 2 describes the observations and the data reduction. The index measurements on published spectroscopy are also presented here. The modelling of intermediate ages SSPs is presented in Sect. 3. In Sect. 4, we discuss the photometric ages for individual clusters and the comparison with the spectroscopically-derived values, while Sect. 5 deals with metallicities and abundance ratios for the star clusters and the diffuse galaxy light. Results are discussed in Sect. 6 and conclusions are drawn in Sect. 7 .

\section{The data}

\subsection{Observations and data reduction}

The $K$-band data were obtained with the Near-Infrared Camera (NIRC, Matthews \& Soifer 1994) on the Keck I telescope on the night of June 20, 1999. The camera operates with a $256 \times 256$ pixels InSb detector, with a pixel size of $30 \mu \mathrm{m}$ corresponding to $0.15^{\prime \prime}$ on the sky. The total field of view is $38^{\prime \prime} \times 38^{\prime \prime}$.

We used a $K_{\mathrm{s}}$ filter for all observations (see Persson et al. 1998 for the transmission curve). We tested various flatfields and finally used one computed from a number of random sky observations done shortly before observing NGC 7252. These were dark subtracted, averaged using a sigma clipping algorithm and normalized. The flatfield was then applied to all science and calibration data.

Two adjacent, slightly overlapping, fields were centered on NGC 7252. The east-west coverage is $\simeq 70^{\prime \prime}$, the northsouth coverage is $38^{\prime \prime}$. The observations were carried out in $300 \mathrm{~s}$ blocks: each block was split into $100 \mathrm{~s}$ ( 5 coadds of $20 \mathrm{~s}$ ) on the east field, $100 \mathrm{~s}$ ( 5 coadds of $20 \mathrm{~s}$ ) on the west field, and $100 \mathrm{~s}$ on the sky (250" north), the latter being divided into 5 dithered exposures ( 5 coadds of $4 \mathrm{~s}$ each). These 5 sky exposures were averaged with a sigma clipping algorithm to create a blank sky field. This sky field (of $20 \mathrm{~s}$ effective exposure time) was normalized to $100 \mathrm{~s}$ effective exposure time and subtracted from both the east and the west field of the same block. The individual images of the east and the west field, obtained this way, were then respectively averaged to compute the final images. The total integration time on each NGC 7252 field was $1300 \mathrm{~s}$. The seeing on the final images, as measured from the FWHM of the objects, was around $0.5^{\prime \prime}$. A composite image of both fields in shown in Fig. 1.

\subsection{K-photometry}

Seven standards from Persson et al. (1998) were observed throughout the night. Most standards were observed more than once at different airmasses. Each standard observation is composed of nine exposures (4 coadds of $1 \mathrm{~s}$ ) in a box pattern. The resulting 144 standard measurements were used to derive a zero point and extinction coefficient; the colour term was assumed to be negligible. We obtained the following relation:

$K_{\mathrm{s}}=24.797( \pm 0.021)+\left(m_{\text {inst }}-0.046( \pm 0.005) \cdot x\right)$, where $m_{\text {inst }}$ is the measured magnitude of an object (normalized to $1 \mathrm{~s}$ exposure) and $x$ is the airmass.

In order to remove any steep background gradients and to perform the photometry on a homogeneous "sky", we modelled the galaxy with a $31 \times 31$ pixels median filter and subtracted it from the original images.

The photometry was performed on the galaxysubtracted images with SExtractor (Bertin \& Arnouts 1996). The detection parameters were set to 5 adjacent pixels $1.5 \sigma$ over the local background (as defined in a $32 \times 32$ pixels box). The photometry was computed in 


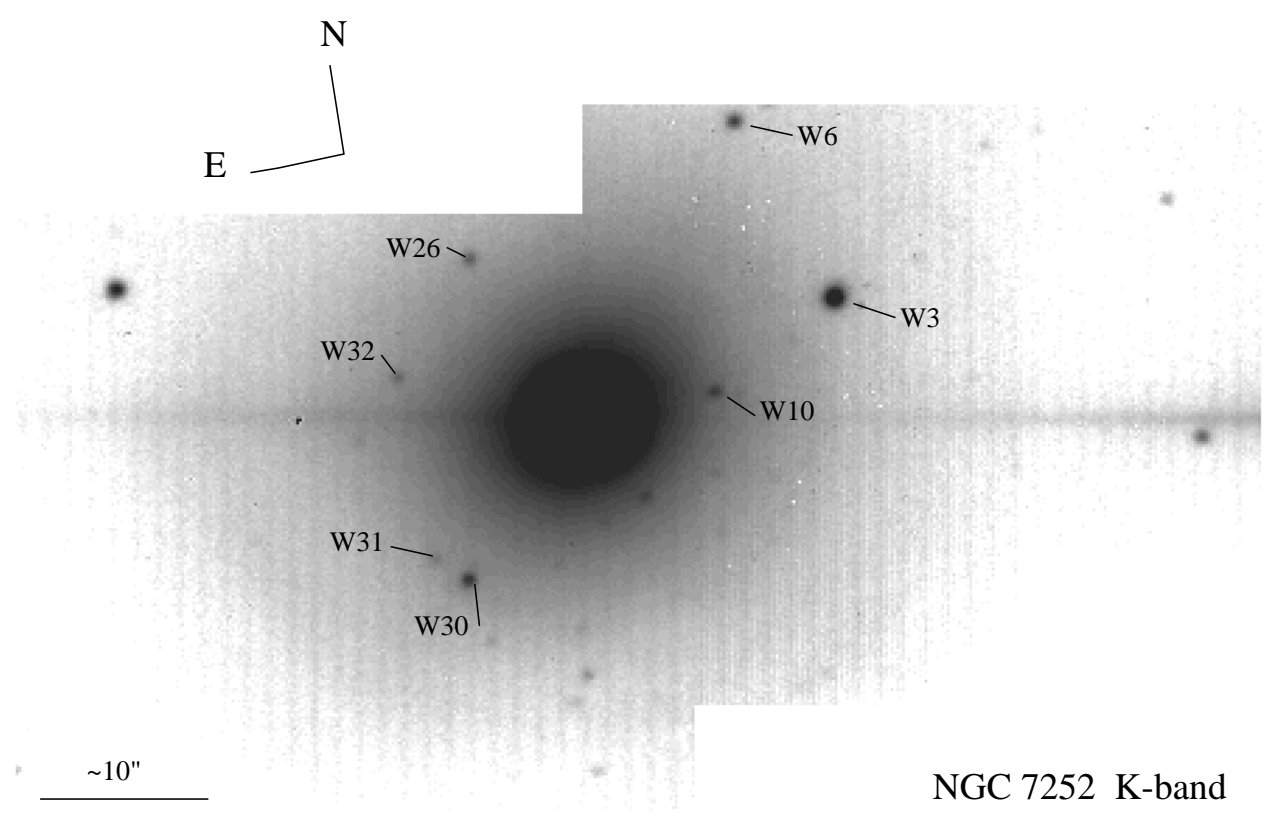

Fig. 1. The composite image of the east and west field. Some clusters are marked as reference, however the contrast chosen for display does not allow us to show all detected clusters

Table 1. $K_{\mathrm{s}}$ magnitudes of young star clusters in NGC 7252. Note that the magnitudes in this table are not corrected for Galactic foreground extinction. The $(V-K)$ colour, however, is corrected for a Galactic foreground extinction of $E(B-V)=$ 0.012

\begin{tabular}{lcc}
\hline Name & $K_{\mathrm{s}}$ & $\left(V-K_{\mathrm{s}}\right)_{0}$ \\
\hline W3 & $15.39 \pm 0.03$ & $2.45 \pm 0.04$ \\
W6 & $17.37 \pm 0.03$ & $2.27 \pm 0.04$ \\
W19 & $20.15 \pm 0.14$ & $2.30 \pm 0.17$ \\
W22 & $19.13 \pm 0.10$ & $2.48 \pm 0.10$ \\
W24 & $20.22 \pm 0.15$ & $2.47 \pm 0.15$ \\
W25 & $19.94 \pm 0.13$ & $2.53 \pm 0.13$ \\
W26 & $18.13 \pm 0.03$ & $2.26 \pm 0.04$ \\
W27 & $20.11 \pm 0.09$ & $1.98 \pm 0.09$ \\
W30 & $17.06 \pm 0.03$ & $2.40 \pm 0.04$ \\
W31 & $19.11 \pm 0.06$ & $1.96 \pm 0.06$ \\
W32 & $18.79 \pm 0.05$ & $2.75 \pm 0.05$ \\
S105 & $19.06 \pm 0.06$ & $\ldots$. \\
S114 & $18.99 \pm 0.08$ & $2.24 \pm 0.09$ \\
\hline
\end{tabular}

different apertures, and finally the "automatic aperture magnitudes" (see Bertin \& Arnouts 1996) were used for consistency with the standard star measurements. The $K_{\mathrm{s}}$ magnitudes were corrected for a Galactic foreground extinction of $E(B-V)=0.012$ (for consistency with Miller et al. 1997, see below), using the relation $A_{K}=$ $0.36 \cdot E(B-V)$ (Fitzpatrick 1999).

The final list of $K_{\mathrm{s}}$ magnitudes (uncorrected for extinction) are given in Table 1 . We followed the naming convention of Schweizer \& Seitzer (1998), mostly referring to the list of Whitmore et al. (1993).
Table 2. Optical magnitudes of young star clusters in NGC 7252 (see references in the text). All values are corrected for a Galactic foreground extinction of $E(B-V)=0.012$

\begin{tabular}{lcccc}
\hline Name & $V_{0}$ & $(U-B)_{0}$ & $(B-V)_{0}$ & $(V-I)_{0}$ \\
\hline W3 & $17.84 \pm 0.02$ & $0.28 \pm 0.06$ & $0.45 \pm 0.04$ & $0.64 \pm 0.04$ \\
W6 & $19.64 \pm 0.02$ & $0.31 \pm 0.06$ & $0.44 \pm 0.04$ & $0.64 \pm 0.03$ \\
W19 & $22.45 \pm 0.10$ & $\ldots$ & $\ldots$. & $0.79 \pm 0.12$ \\
W22 & $21.61 \pm 0.02$ & $0.20 \pm 0.08$ & $0.44 \pm 0.04$ & $0.68 \pm 0.04$ \\
W24 & $22.69 \pm 0.03$ & $0.35 \pm 0.17$ & $0.41 \pm 0.06$ & $0.69 \pm 0.05$ \\
W25 & $22.47 \pm 0.02$ & $0.57 \pm 0.16$ & $0.36 \pm 0.05$ & $0.63 \pm 0.04$ \\
W26 & $20.39 \pm 0.02$ & $0.31 \pm 0.06$ & $0.53 \pm 0.04$ & $0.68 \pm 0.03$ \\
W27 & $22.09 \pm 0.02$ & $0.37 \pm 0.10$ & $0.38 \pm 0.04$ & $0.69 \pm 0.04$ \\
W30 & $19.46 \pm 0.02$ & $0.26 \pm 0.06$ & $0.41 \pm 0.04$ & $0.63 \pm 0.03$ \\
W31 & $21.07 \pm 0.02$ & $0.27 \pm 0.07$ & $0.29 \pm 0.04$ & $0.54 \pm 0.03$ \\
W32 & $21.54 \pm 0.02$ & $0.97 \pm 0.18$ & $0.80 \pm 0.04$ & $1.08 \pm 0.04$ \\
S105 & $\ldots$. & $\ldots$ & $\cdots$ & $\cdots$ \\
S114 & $21.23 \pm 0.03$ & $0.32 \pm 0.09$ & $0.42 \pm 0.06$ & $0.62 \pm 0.05$ \\
\hline
\end{tabular}

\subsection{Previous optical and UV photometry}

The UV and optical photometry is taken from Miller et al. (1997), except for object S114 for which we used the photometry given by Schweizer \& Seitzer (1998), and for object W19, for which we used the photometry given in Whitmore et al. (1993). There is no optical photometry available for object S105.

The magnitudes used in our work are given in Table 2 . All magnitudes are corrected for Galactic foreground extinction adopting $E(B-V)=0.012$, following Miller et al. (1997). 
We note that Miller et al. (1997) give a warning to potential users of their $U$-band photometry for which they note, among other problems, a 0.16 magnitude offset when compared to ground-based work. Therefore we refrain from using the $(U-B)$ colours in our photometric analysis. The $B, V$ and $I$ photometry is unaffected by problems.

\subsection{Spectroscopy}

To date, the only database of spectroscopic observations of young star clusters in NGC 7252 has been provided by Schweizer \& Seitzer (1998).

The authors present ultraviolet-to-visual spectra for eight objects of the outer blue population, namely W3, W6, W26, W30, W31, S105, S114, S101. With the exception of S101, whose spectrum is dominated by emission lines due to a surrounding giant H II region, all the spectra are characterized by strong Balmer lines $(\mathrm{H} \beta \sim 6-13 \AA$ ) in absorption (see Fig. 2). These features are used by Schweizer \& Seitzer (1998) to determine the ages of the clusters, by comparison with a version of the models by Bruzual \& Charlot (1996, quoted as private communication) computed with the needed high resolution of the observed spectra $(\lambda \lesssim 2 \AA)$. This restricts their comparisons to solar metallicity models, because of the lack of high-resolution model spectra for other metallicities. In the next section we present new measurements of the Balmer lines for the cluster sample of Schweizer \& Seitzer (1998). These will be compared with our model spectra, on which the ages determined from photometry are based (see Sect. 4).

\subsection{The new index measurements}

The cluster spectra by Schweizer \& Seitzer (1998, courtesy of F. Schweizer, private communication) are used as a complement to our photometric analysis (see Sect. 4). In order to allow a sensible comparison, observed and model spectra must have the same resolution, and the strengths of relevant absorption features must be measured exactly in the same way on the observed and the synthetic spectrum. Therefore the spectra of Schweizer \& Seitzer (1998) have been broadened to match the resolution of $20 \AA$ (in the visual region) of the model spectra.

The equivalent widths $(E W \mathrm{~s})$ of the Balmer lines $\mathrm{H} \beta$, $\mathrm{H} \gamma$ and $\mathrm{H} \delta$ are then measured adopting the definitions given in Brodie et al. (1998, LS indices, their Table 3), designed to study young stellar populations. The bandpass definitions are similar to the ones given in Bruzual \& Charlot (1993), but with a different choice for the continuum. The flux in the continuum for the LS indices is determined as the average flux in the continuum bandpasses while it coincides with the flux at the end points of the continuum bandpasses in Bruzual \& Charlot (1993). Therefore the LS indices are better suited to spectra with low $S / N$. They agree well with the Bruzual \& Charlot
Table 3. Equivalent widths of Balmer lines $\mathrm{H} \beta, \mathrm{H} \gamma$ and $\mathrm{H} \delta$, for star clusters in NGC 7252 (spectra from F. Schweizer). An indicative value of the signal-to-noise ratio per $\AA$ is given in the last column

\begin{tabular}{lcccc}
\hline Name & $\mathrm{H} \beta(\AA)$ & $\mathrm{H} \gamma(\AA)$ & $\mathrm{H} \delta(\AA)$ & $S / N$ \\
\hline W3 & $10.1 \pm 0.3$ & $9.1 \pm 0.1$ & $12.2 \pm 0.5$ & $\sim 20$ \\
W6 & $9.1 \pm 0.6$ & $8.5 \pm 0.6$ & $11.7 \pm 0.3$ & $\sim 8$ \\
W26 & $7.3 \pm 2.9$ & $8.3 \pm 2.2$ & $13.8 \pm 1.5$ & $\sim 5$ \\
W30 & $11.7 \pm 1.0$ & $9.3 \pm 1.2$ & $12.0 \pm 0.3$ & $\sim 8$ \\
W31 & $6.2 \pm 3.5$ & $6.9 \pm 3.8$ & $12.5 \pm 5.8$ & $\sim 3$ \\
S105 & $9.0 \pm 2.0$ & $12.7 \pm 3.0$ & $4.7 \pm 2.7$ & $\sim 3$ \\
S114 & $5.4 \pm 1.1$ & $8.2 \pm 1.6$ & $9.5 \pm 5.4$ & $\sim 3$ \\
\hline
\end{tabular}

ones for spectra with high signal-to-noise (see Brodie et al. 1998 for further details). We chose both for the modelled spectra and the observed ones to measure the $E W \mathrm{~s}$ by centering the index definitions on the absorption feature, in order to circumvent possible uncertainties in the rest frame corrections of the spectra. Minimal corrections are necessary and do not significantly affect the $E W$ measurements.

The resulting Balmer line $E W \mathrm{~s}$ are given in Table 3. The quoted errors were taken from Schweizer \& Seitzer (1998). Since we did not have the raw spectra, we were not able to estimate errors based on photon noise. A reference value of the signal-to-noise ratio, as determined by a visual inspection of the spectra, is listed in the last column.

It is worth mentioning that for strong, well-defined absorption features the broadening procedure does not imply loss of information. For comparison, we compute the same $E W$ s on the original ( $\sim 2 \AA$ resolution) spectra from Schweizer \& Seitzer (1998). On average, the higher resolution implies larger $E W \mathrm{~s}$, the difference being very small $(\sim 0.08 \AA)$ for high $S / N$ spectra (like W3), and as large as $\sim 4 \AA$ for poorly observed spectra (like S105).

The EWs in Table 3 can be compared to those in Table 3 of Schweizer \& Seitzer (1998), bearing in mind that the latter refer to different bandpasses and continuum levels, and are measured on the $\sim 2 \AA$ resolution spectra. The comparison shows that the relative strenghts of Balmer lines for a specific cluster, are the same as in Schweizer \& Seitzer (1998). Also, the relative strenghts of Balmer lines of the cluster sample are consistent, with W3 and W30 exhibiting the strongest Balmer lines. The values in our Table 3 will be used in Sect. 4.3 to derive spectroscopic cluster ages.

\section{The AGB phase transition in intermediate-age stellar populations}

The first development in a stellar population of stars with degenerate carbon-oxygen cores is the appearance of an extended hydrogen and helium shell-burning phase close to the Hayashii line, called the Asymptotic Giant Branch (AGB). The AGB phase consists of an early period (Early 


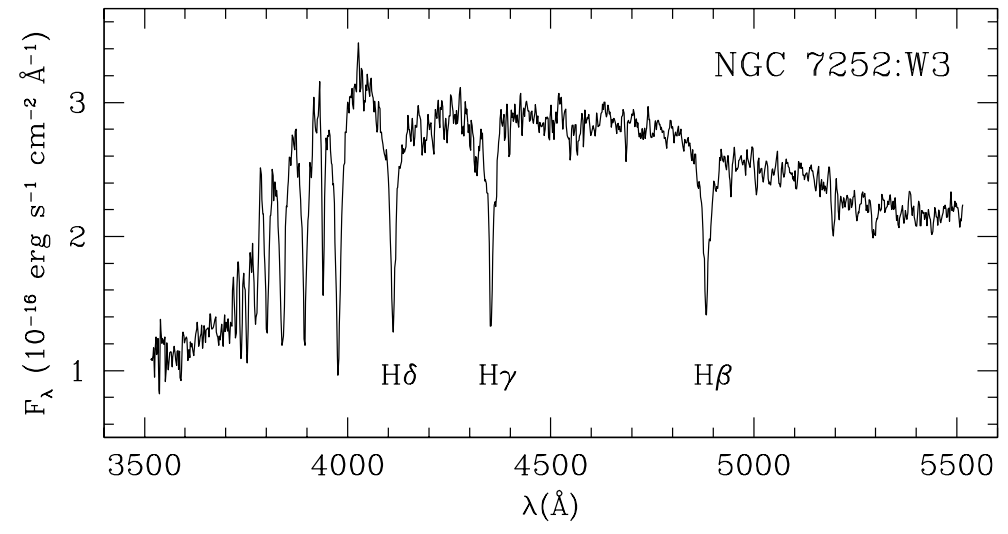

Fig. 2. Ultraviolet-to-visual spectrum of cluster W3 in NGC 7252, from Schweizer \& Seitzer (1998). Note the strong Balmer lines in absorption

AGB, E-AGB), in which the hydrogen-burning shell is quiescent, and a subsequent, longer stage in which both shells are active (Thermally Pulsing AGB, TP-AGB). The so-called AGB phase-transition (Renzini 1981; Renzini \& Buzzoni 1986) corresponds to the epoch at which a large amount of fuel is burned during the TP-AGB phase. As a consequence, a sharp increase of the total bolometric and infrared luminosity of the population is expected. The AGB phase transition starts at ages 200-300 Myr (see Iben \& Renzini 1983; Renzini \& Voli 1981) lasts less than $\sim 1$ Gyr and has sizable effects on the spectral energy distribution of the population.

The inclusion of the TP-AGB contribution in population synthesis studies is complicated by the poor knowledge of the real mechanisms driving the evolution (see Girardi \& Bertelli 1998 for a thorough investigation). Uncertainties in mass loss, mixing, and efficiency of hydrogen burning at the bottom of the convective envelope (known as the envelope burning process) together prevent pure theory from predicting the amount of fuel (see Sect. 3.1) burned during this phase (Renzini \& Buzzoni 1986). Star clusters in the Magellanic Clouds cover the interesting range in ages and offer the opportunity to calibrate these effects.

Maraston (1998) presents models for Simple Stellar Populations (SSPs) in which the TP-AGB contribution to the total energy as a function of age is calibrated with the observed contribution in Magellanic Cloud clusters. These SSP models simultaneously match the optical and infrared colours of the intermediate age clusters. Unfortunately the AGB phase transition in the Magellanic Cloud clusters is the only observational counterpart of theoretical computations, allowing the calibration of models with metallicity $\sim 0.5 Z_{\odot}$. The dependence on $Z$ may be taken into account using the theoretical prescriptions of Renzini \& Voli (1981). This is explored in Maraston (1998) for solar metallicity models. For this work we compute intermediate age SSPs models for various metallicities taking as a basis the calibration by Maraston (1998) and adopting the prescriptions by Renzini \& Voli (1981) to describe the influence of $Z$ on the AGB phase. The next section reports the adopted procedure in more detail.

\subsection{Modelling the different chemical compositions}

For the present work, new sets of SSP models with metallicities $0.5 Z_{\odot}([\mathrm{Fe} / \mathrm{H}]=-0.33)$ and $2 Z_{\odot}([\mathrm{Fe} / \mathrm{H}]=0.35)$, and ages $t \geq 55 \mathrm{Myr}$ and $t \geq 100 \mathrm{Myr}$ respectively, are computed with the evolutionary population synthesis code described in Maraston (1998). The code is based on the fuel consumption theorem (Renzini \& Buzzoni 1986) which allows a correct evaluation of the energetics of the post Main Sequence phases. The fuel consumption theorem states that the contribution of any post Main Sequence phase "j" to the total luminosity, $L_{\mathrm{j}}^{\mathrm{bol}}(t, Z)$, is proportional to the amount of fuel burned in that phase, $F_{\mathrm{j}}$, through the relation

$L_{\mathrm{j}}^{\mathrm{bol}}(t, Z)=9.7510^{10} b(t, Z) F_{\mathrm{j}}(t, Z)$.

$b(t, Z)$, the "evolutionary flux", is the rate of evolution off the Main Sequence, the fuel $F_{\mathrm{j}}$ is the amount of hydrogen and /or helium (in $M_{\odot}$ ) burned during the phase "j" by the evolutionary mass corresponding to the age $t$ of the population, which is the turn-off mass $M_{\mathrm{TO}}(t, Z)$.

The input stellar tracks from the dwarf Main Sequence up to the end of the E-AGB phase, are from Bono et al. (1997) and S. Cassisi (1999, private communication). Synthetic stellar spectra as functions of effective temperature, gravity and metallicity, for $T_{\mathrm{e}} \gtrsim 3500 \mathrm{~K}$, are taken from the spectral library compiled by Lejeune et al. (1998). Empirical colours for C-and M-type AGB stars are taken from various sets of observational data in the Magellanic Clouds, as described in Maraston (1998). A wider discussion of the complete set of new SSP models is given in Maraston (2001, in preparation). Here we concentrate on the intermediate-age SSPs used in the present analysis. 


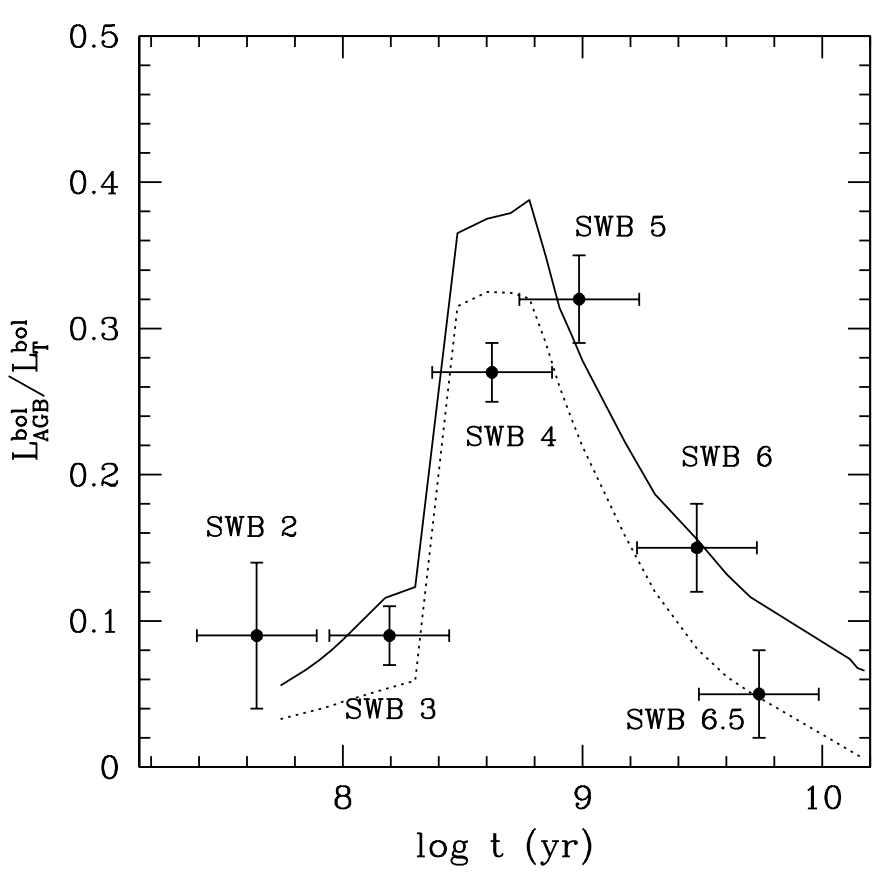

Fig. 3. The synthetic AGB contribution to the total bolometric luminosity compared with the LMC globular cluster data from Frogel et al. (1990). The contribution from the TP-AGB alone is separately shown as a dotted line

The Initial Mass Function (IMF) of these models, unless explicitly stated, is a power-law with Salpeter exponent over the whole mass range $\left(0.1-100 M_{\odot}\right)$, because the impact of the exact exponent of the IMF on broadband colours is small, particularly for young stellar systems (see Maraston 1998).

The fuel consumption during the TP-AGB phase as a function of metallicity is computed on the basis of the calibrated TP-AGB fuel for $Z=Z_{\odot}$ (Maraston 1998). The adopted procedure is as follows. For a population of age $t$ and metallicity $Z$, the TP-AGB fuel is a fraction of the envelope mass at the onset of the TP-AGB phase, $M_{\mathrm{env}}^{\mathrm{TP}-\mathrm{AGB}}(t, Z)$, that in turn depends on the present core mass. Both quantities are extracted from the appropriate evolutionary track. The calibrated fuel $F^{\mathrm{TP}-\mathrm{AGB}}(t)$ for $Z=Z_{\odot}$ given in Table 1 of Maraston (1998) allows the computation of the fraction of fuel burned as a function of $M_{\mathrm{env}}^{\mathrm{TP}-\mathrm{AGB}}(t)$ for $Z=Z_{\odot}$. The metallicity dependence of the envelope mass is known. Interpolation in $M_{\mathrm{env}}^{\mathrm{TP}}-\mathrm{AGB}(t, Z)$ then allows the evaluation of the TPAGB fuel for various metallicities, $F^{\mathrm{TP}-\mathrm{AGB}}(t, Z)$.

The core mass, and therefore the envelope mass $M_{\mathrm{env}}^{\mathrm{TP}-\mathrm{AGB}}$ at the onset of the TP-AGB phase, do not strongly depend on metallicity for any initial mass. This effect together with the Magellanic cluster calibration, results in a total TP-AGB fuel which is fairly independent of metallicity. Instead the metal content plays a role in determining the spectral type of the stars burning the total fuel, as will be explained later. Figure 3 shows the calibration of the synthetic AGB contribution to the total bolometric luminosity as a function of age, evaluated with the fuel consumption theorem. The data points in Fig. 3 are the observed AGB contributions in Magellanic Cloud clusters (data from Frogel et al. 1990), grouped in age bins adopting the classification scheme of Searle et al. (1980, SWB). Within each bin, the luminosities of all the clusters' AGB stars are added, and then divided by the sum of all the integrated luminosities of the same clusters. This procedure minimizes the large stochastic fluctuations (due to the sparsely populated AGBs) between clusters having a same SWB type (i.e. age). The AGB phase transition is observed among the SWB groups $3-5$. The figure is the analogue of Fig. 3 in Maraston (1998) except that the data are now compared with models of $Z=0.5 Z_{\odot}$, more appropriate for Magellanic Cloud clusters.

The next step in computing realistic colours for SSPs is calibrating the distribution of the total TP-AGB fuel among the various effective temperatures. The TP-AGB phase is populated by stars of spectral type $\mathrm{C}$ (carbon stars) and $\mathrm{M}$, the production of $\mathrm{C}$ stars being a function of both the initial stellar mass (hence of the SSP age) and the metallicity (cf. Renzini \& Voli 1981). From the data for Large Magellanic Cloud clusters (Frogel et al. 1990, Table 4), Maraston (1998) evaluated the contributions of $\mathrm{C}$ and $\mathrm{M}$ stars to the bolometric light for each age bin. The calibration, reported in Table 2 and Fig. 4 of Maraston (1998), shows that C-stars characterized intermediateage SSPs (0.2-2 Gyr). Very old stellar populations (e.g. Galactic globular clusters and the Galactic Bulge) do not show any evidence for C-stars, in agreement with the theoretical expectations. The very red infrared colours of the intermediate age Magellanic Cloud clusters are due to the presence of luminous $\left(M_{\mathrm{bol}}<-4\right)$ carbon stars, which are absent in the youngest and oldest clusters and have no effect on visible colours (Persson et al. 1983).

The initial metal content $Z$ of the stellar population plays a key role in determining the production and the characteristics of carbon stars (Renzini \& Voli 1981). By decreasing $Z$, the abundance of oxygen in the envelope is lower, thus a lower amount of carbon has to be dredgedup in order to achieve $\mathrm{C} / \mathrm{O}>1$ and create carbon stars. Therefore, a metal poor stellar population is expected to produce many more carbon stars than a metal rich one of the same age. Increasing $Z$ from $\sim 0.5 Z_{\odot}$ (appropriate for LMC) to $2 Z_{\odot}$ reduces the fraction of carbon stars by a factor of $\sim 4$ (Renzini \& Voli 1981; see also Marigo 1999). This scaling has been adopted to distribute the fuel among C- and M-stars, as a function of metallicity. Figure 4 shows the resulting time evolution of the $B-V$ and $V-K$ colours for SSPs with metallicity $0.5 Z_{\odot}, Z_{\odot}$ and $2 Z_{\odot}$. Colours in other bands will be presented in Maraston (2001, in preparation).

As already shown in Maraston (1998), the AGB phase transition has a sizeable effect on SSP infrared colours. To give an idea of this effect, an $\operatorname{SSP}$ (with $Z=0.5 Z_{\odot}$ ) is computed without including the TP-AGB contribution (dot-dashed lines). This models also shows that, as expected, the effect of AGB stars on optical colours is negligible because these stars mainly radiate in the infrared. 


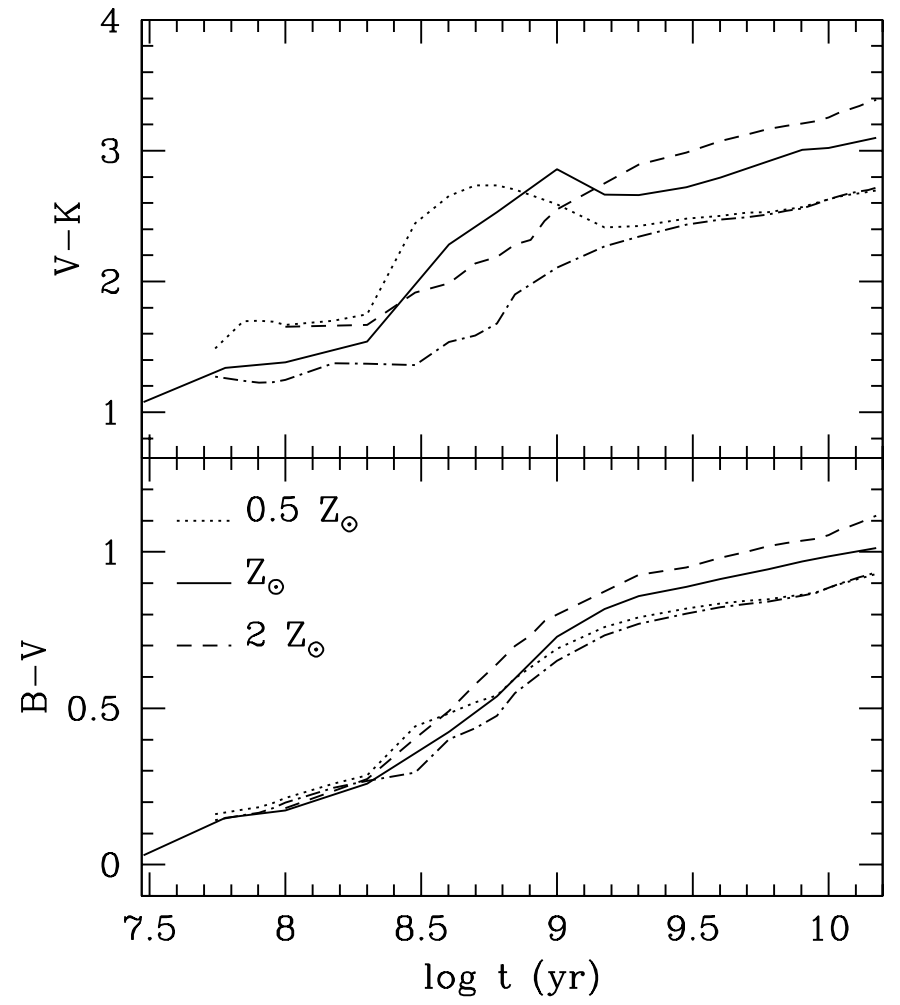

Fig. 4. Time evolution of $B-V$ (lower panel) and $V-K$ (upper panel) for SSPs of various metallicities (labelled in the lower panel). The dot-dashed lines are models with $Z=0.5 Z_{\odot}$ in which the TP-AGB contribution is not taken into account

Note that the impact of the AGB phase transition decreases with the increasing metallicity of the SSP in our modelling. This is because less fuel is burned by C-like stars in a metal-rich population. In Sect. 4.1 we show that models from other authors do not reproduce this trend.

The SSP models displayed in Fig. 4 are used to determine the ages of the star clusters of NGC 7252, as shown in the next section.

\section{The age distribution of NGC 7252 clusters}

\subsection{Evidence for the $A G B$ phase-transition}

The evolution of SSP optical colours, such as $B-V$, is monotonic with age (see Fig. 4) because these mainly trace the evolution of the turnoff region of the population. As will be shown in the next section, the Balmer line strengths in contrast, have a maximum at about 300 Myr (cf. Fig. 8). Therefore optical colours help greatly in constraining the age of young SSPs. For reasons discussed in Sect. 3, an optical-to-infrared colour like $V-K$ is particularly useful for studying intermediate-age populations dominated by AGB stars. Furthermore, $V-K$ has the advantage of being calibrated by the observed colours of LMC clusters, due to the many sets of $K$-band observations that are available (see Maraston 1998 and reference therein).

Figure 5 shows $V-K$ vs. $B-V$ for the star clusters in NGC 7252 (values from Tables 1 and 2). Also shown are data for the intermediate-age GCs in Magellanic Clouds (open triangles, references in the caption). The arrow shows the total Galactic foreground + internal extinction vector; we refer to Sect. 4.4 where the negligible impact of reddening on our age determination is discussed. The synthetic relations for the SSPs shown in Fig. 4 are overplotted. Some reference ages are indicated for the $Z=Z_{\odot}$ model.

Figure 5 clearly shows the AGB phase transition among the young star clusters in NGC 7252. Both the optical and infrared colours of the observed clusters are well described by our SSP models. The observed range in infrared colours is not recovered if the TP-AGB contribution is not taken into account in the synthesis (dot-dashed line model). In the framework of the assumptions on the metallicity dependence of the TP-AGB fuel (Sect. 3), halfsolar or solar metallicities are favoured for these GCs. In Sect. 5 a more insightful discussion on the metallicities of selected clusters is given.

The young GCs of NGC 7252 lie in the narrow age range 200 Myr to 500 Myr, clustering around $\sim 300$ Myr. An important exception is object W32 $(B-V \sim 0.8$ in Fig. 5), which seems to have already completed the AGB phase transition. The existence of such an old cluster would impact on the assumed duration of the star formation process triggered by merging (see Sect. 6).

As discussed in Sect. 3, the treatment of the TP-AGB phase in population synthesis represents a major difficulty, and intermediate-age SSPs models from various authors show sizeable discrepancies (see Maraston 1998). Figure 6 shows the $V-K$ vs. $B-V$ diagrams for the SSP models of Bruzual \& Charlot (2000, private communication, upper panel), and Tantalo et al. (1996, lower panel), with metallicities indicated in the upper panel. The data are the same as in Fig. 5. The trend of the synthetic $V-K$ with $Z$, for intermediate age SSPs is very different in the various models. The Tantalo et al. (1996) models are redder at increasing metallicity and do not show evidence for the AGB phase transition in $V-K$. As already noted in Maraston (1998), the jump in $V-K$ shown by the Tantalo et al. (1996) models corresponds to very blue $B-V(\sim 0.15 \mathrm{mag})$ colours, and is in strong disagreement with the observations. The models by Bruzual and Charlot scale similarly with metallicity, predicting redder colours with increasing $Z$. However these models are much bluer than those of Tantalo et al. (1996) and do not show an appreciable difference between the solar and half-solar metallicity cases. We note that the infrared luminosity of intermediate age SSPs in the most recent version of the Bruzual \& Charlot models is lower than the in Bruzual \& Charlot (1996) models. This worsens the comparison with the LMC data shown in Maraston (1998). In the Bruzual \& Charlot models shown here, intermediate-age LMC GCs with $B-V>0.4$ are recovered by the $2.5 Z_{\odot}$ SSP, overestimating the real metallicity of these clusters by a factor of $\sim 5$. The GCs of NGC 7252 (filled dots) are of intermediate age, with $Z \simeq 2.5 Z_{\odot}$ according to BC2000 models, 


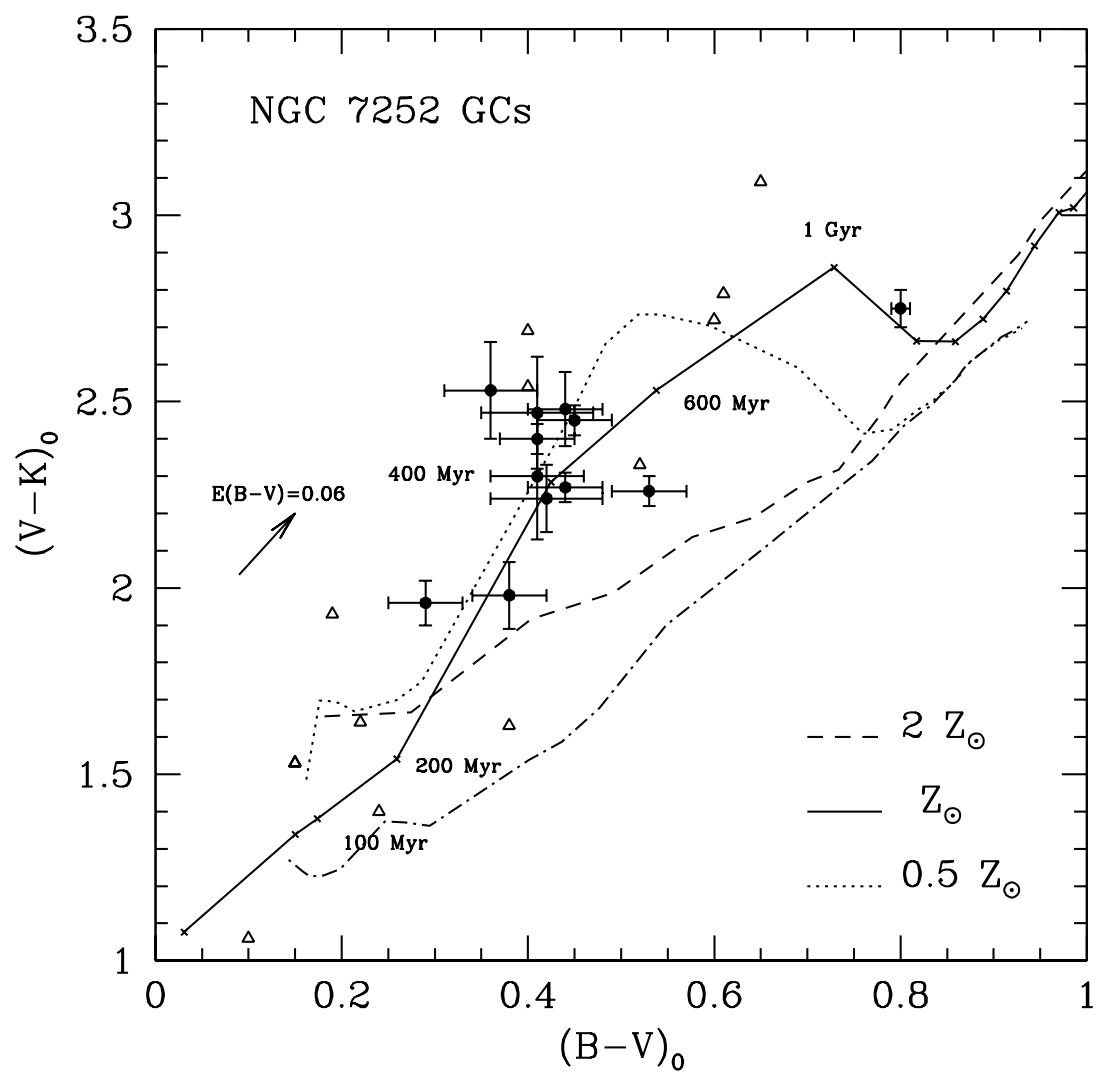

Fig. 5. The $V-K$ vs. $B-V$ diagram for the star clusters in NGC 7252 (filled circles). Also shown are LMC intermediate-age $(3 \lesssim S W B \lesssim 5.5)$ GCs (open triangles), with $B-V$ from van den Bergh (1981) and $V-K$ from Persson et al. (1983). SSP models for various metallicities are over-plotted. The dot-dashed line is a model with $Z=0.5 Z_{\odot}$ in which the TP-AGB contribution is not taken into account

and are of intermediate age, with $Z \lesssim 0.5 Z_{\odot}$ according to the models by Tantalo et al. (1996).

\subsection{Ages from photometry}

The ages for the individual NGC 7252 clusters are obtained by interpolating the model colours (and ages) shown in Fig. 4 for the observed $B-V$ and $V-K$ colours (Tables 1 and 2), separately.

The results are reported in Table 4, for metallicities $0.5,1,2 Z_{\odot}$. The uncertainties on the ages reflect the photometric errors given in Tables 1 and 2. If the values are preceded by the $\sim$ symbol, the observed colour is larger (or smaller) than the maximum (minimum) model colour. In this case, no extrapolations are made. The quoted age corresponds to the model which is closest to the observed colour.

The last column gives the age derived by Schweizer \& Seitzer (1998) from the HST $V-I$ photometry of Miller et al. (1997), using the 1996 release of the Bruzual \& Charlot models with solar metallicity. The ages from Schweizer \& Seitzer (1998) agree well with ours, as determined from $B-V$ (for solar metallicity).

The comparison between the ages from $B-V$ and $V-K$ given in Table 4 is shown in Fig. 7 for the various metallicities. The models with $Z=0.5 Z_{\odot}$ provide the best agreement between the ages derived from $B-V$ and the ones derived from $V-K$. For some clusters, solar metallicity models (central panel) also provide a good match (cf. Table 4). Our models with a higher metallicity $\left(Z=2 Z_{\odot}\right.$, upper panel) fit the data significantly less well. In fact, due to the lower amount of fuel burned by carbon stars at increasing metallicity (see Sect. 3), infrared colours are bluer for a given optical colour and the ages derived from $V-K$ are systematically larger than the ones derived from $B-V$. We note again that, due to the lack of calibrations for the theoretical predictions, conclusions on the metallicities of these clusters are only tentative.

As a final remark, it is important to note that the photometric ages of Table 4 do not depend on the particular choice of the Salpeter IMF because, as anticipated in Sect. 3.1, broadband colours are fairly insensitive to the IMF slope. To quantitatively explore the effect of the IMF, we consider an IMF biassed towards large masses with exponent -1.5 (in the notation where Salpeter is -2.35) and metallicity $Z=0.5 Z_{\odot}$. The impact on broad-band colours and mass-to-light ratios of an IMF of this kind is discussed in Maraston (1998). Recalling the results, the optical and IR colours of a giant-dominated SSP are redder than those of a Salpeter one for ages $\lesssim 4$ Gyr, the maximum difference being $\sim 0.04 \mathrm{mag}$ and $\sim 0.12 \mathrm{mag}$ for $B-V$ and $V-K$ respectively. For ages older than 4 Gyr, these 
Table 4. Photometric cluster ages (in Myr) determined on the SSPs with metallicities indicated in the first line. The uncertainties reflect the photometric errors. The last column contains the photometric age derived by Schweizer \& Seitzer (1998) using $V-I$ for $Z=Z_{\odot}$

\begin{tabular}{lccccccc}
\hline & \multicolumn{2}{c}{$0.5 Z_{\odot}$} & \multicolumn{2}{c}{$Z_{\odot}$} & \multicolumn{2}{c}{$2 Z_{\odot}$} & $Z_{\odot}$ \\
Name & $B-V$ & $V-K$ & $B-V$ & $V-K$ & $B-V$ & $V-K$ & $V-I$ (Schweizer \& Seitzer 1998) \\
\hline W3 & $320 \pm 70$ & $300 \pm 10$ & $440 \pm 60$ & $530 \pm 30$ & $350 \pm 50$ & $890 \pm 30$ & $420 \pm 110$ \\
W6 & $300 \pm 60$ & $270 \pm 10$ & $420 \pm 60$ & $390 \pm 20$ & $340 \pm 50$ & $690 \pm 70$ & $420 \pm 90$ \\
W19 & - & $280 \pm 30$ & - & $410 \pm 100$ & - & $750 \pm 200$ & - \\
W22 & $300 \pm 60$ & $300 \pm 40$ & $420 \pm 60$ & $550 \pm 90$ & $340 \pm 40$ & $920 \pm 110$ & - \\
W24 & $280 \pm 60$ & $300 \pm 50$ & $380 \pm 90$ & $540 \pm 130$ & $310 \pm 60$ & $910 \pm 170$ & - \\
W25 & $240 \pm 30$ & $340 \pm 60$ & $300 \pm 60$ & $600 \pm 120$ & $260 \pm 40$ & $970 \pm 190$ & - \\
W26 & $550 \pm 120$ & $270 \pm 10$ & $580 \pm 70$ & $390 \pm 20$ & $440 \pm 50$ & $670 \pm 60$ & - \\
W27 & $260 \pm 30$ & $230 \pm 10$ & $330 \pm 60$ & $300 \pm 30$ & $280 \pm 40$ & $390 \pm 80$ & $400 \pm 90$ \\
W30 & $280 \pm 30$ & $290 \pm 10$ & $380 \pm 60$ & $480 \pm 30$ & $310 \pm 40$ & $850 \pm 30$ & $230 \pm 40$ \\
W31 & $200 \pm 40$ & $230 \pm 10$ & $230 \pm 40$ & $300 \pm 20$ & $210 \pm 40$ & $360 \pm 60$ & - \\
W32 & $2300 \pm 300$ & $\sim 2000$ & $1400 \pm 60$ & $1250 \pm 130$ & $1000 \pm 50$ & $1460 \pm 140$ & $370 \pm 110$ \\
S114 & $280 \pm 70$ & $270 \pm 10$ & $390 \pm 90$ & $380 \pm 40$ & $320 \pm 60$ & $650 \pm 140$ & - \\
\hline
\end{tabular}

colours become bluer than those for a Salpeter IMF by $\sim 0.01 \mathrm{mag}$ and $\sim 0.06 \mathrm{mag}$ respectively. The photometric ages derived from the SSP with a giant-dominated IMF are lower by $\sim 20-30 \mathrm{Myr}$ (e.g. for W3 we obtain an age of $\sim 280 \mathrm{Myr}$ ) than the ones derived from the SSP with a Salpeter IMF. The effect of the IMF is therefore negligible for the purposes of age determination.

\subsection{Photometric versus spectroscopic ages}

It is interesting to check how the ages derived from photometry compare to the ones spectroscopically determined in the framework of the same modelling. With this aim the strengths of the Balmer lines $\mathrm{H} \beta, \mathrm{H} \gamma$ and $\mathrm{H} \delta$ were measured on the spectra of the SSP models described in Sect. 3.1. The same procedure (spectral resolution and bandpass definitions) was adopted as was used for the observed spectra (Sect. 2.5, Table 3).

The results are given in Table 5 . The ages are obtained by interpolating the model $E W \mathrm{~s}$ (and ages) for the various metallicities (given in the first line) for the observed $E W \mathrm{~s}$. In case of two possible solutions for the age (cf. Fig. 8), the age derived from the photometry is used to choose between the two options. As for Table 4, if the values are preceded by the $\sim$ symbol, the observed $E W$ is larger (or smaller) than the maximum (minimum) model $E W$. In this case, the quoted age corresponds to the model which is closest to the observed $E W \mathrm{~s}$. In order to guide the comparison, a value for the average Balmer age $\left(t_{\mathrm{av}}\right)$ is also given in Table 5. This is simply obtained by averaging the values derived from the three Balmer lines. The approximate values $(\sim)$ are not taken into account in the average. The uncertainties are computed using only the errors on the $E W$ s given by Schweizer \& Seitzer (1998) (see Table 3), and not taking into account any systematic errors in the models.
The comparison between the spectroscopic and the photometric ages (Table 4) is shown in Fig. 8.

Spectroscopic and photometric ages are consistent when the symbols lie inside the shaded regions. Note that the object S105, for which we do not have photometry, is not included in the comparison of Fig. 8. In general, the average spectroscopic ages are in good agreement with the ones derived from photometry, within the large errors affecting most of the spectra. Because of this, the photometry greatly helps in constraining the age for poorly observed spectra. An example is object S114, for which Schweizer \& Seitzer (1998) could not assess the age.

\subsection{Internal reddening}

Our infrared photometry (Table 1) and the optical photometry from the literature (Table 2) are corrected for a Galactic foreground extinction of $E(B-V)=0.012$ (see Sects. 2.2 and 2.3). In this section we explore the impact of internal reddening on the cluster ages derived from photometry.

NGC 7252 shows evidence for dust in the central region $(r \lesssim 2.5 \mathrm{kpc})$, associated with spiral-arm like structures (Whitmore et al. 1993; Miller et al. 1997). As discussed in these studies, most of the clusters belonging to the "outer" galaxy region $\left(6^{\prime \prime}\right.$ to $\left.\sim 120^{\prime \prime}\right)$, are not found in dusty regions. Therefore it is likely that our magnitudes are not severely affected by internal reddening. Moreover, the IUE spectrum of NGC 7252 is rather blue, which is also indicative that the actual internal reddening is likely to be not too large (D. Calzetti, private communication). From the Third Reference Catalog of Bright Galaxies (RC3, de Vaucouleurs et al. 1991) the global $E(B-V)$ is $\sim 0.06$. This estimate contains the differential Galactic foreground extinction as determined by Burstein \& Heiles (1984), and 
Table 5. Spectroscopic cluster ages (in Myr). Metallicities of the models are given in the first line. Uncertainties are computed using the errors given by Schweizer \& Seitzer (1998)

\begin{tabular}{lcccccccc}
\hline & \multicolumn{9}{c}{$0.5 Z_{\odot}$} & \multicolumn{4}{c}{$Z_{\odot}$} \\
Name & $\mathrm{H} \beta$ & $\mathrm{H} \gamma$ & $\mathrm{H} \delta$ & $t_{\mathrm{av}}$ & $\mathrm{H} \beta$ & $\mathrm{H} \gamma$ & $\mathrm{H} \delta$ & $t_{\mathrm{av}}$ \\
\hline W3 & $\sim 400$ & $250 \pm 10$ & $\sim 400$ & $250 \pm 10$ & $420 \pm 20$ & $600 \pm 10$ & $\sim 400$ & $510 \pm 10$ \\
$\mathrm{~W} 6$ & $310 \pm 70$ & $200 \pm 90$ & $\sim 400$ & $260 \pm 80$ & $600 \pm 90$ & $640 \pm 40$ & $430 \pm 30$ & $560 \pm 50$ \\
W26 & $800 \pm 600$ & $710 \pm 200$ & $\sim 400$ & $760 \pm 400$ & $780 \pm 480$ & $650 \pm 170$ & $\sim 400$ & $710 \pm 320$ \\
W30 & $\sim 400$ & $270 \pm 100$ & $\sim 400$ & $270 \pm 100$ & $\sim 400$ & $580 \pm 140$ & $\sim 400$ & $580 \pm 140$ \\
W31 & $\sim 60$ & $\sim 60$ & $\sim 400$ & - & $\sim 30$ & $30 \pm 300$ & $\sim 400$ & $30 \pm 300$ \\
S114 & $\sim 60$ & $180 \pm 180$ & $220 \pm 200$ & $200 \pm 190$ & $\sim 30$ & $110 \pm 130$ & $140 \pm 1000$ & $130 \pm 560$ \\
\hline
\end{tabular}

\begin{tabular}{lcccc}
\hline & \multicolumn{2}{c}{$2 Z_{\odot}$} & \multicolumn{2}{c}{ Schweizer \& Seitzer $(1998)\left(Z_{\odot}\right)$} \\
$\mathrm{H} \beta$ & $\mathrm{H} \gamma$ & $\mathrm{H} \delta$ & $t_{\mathrm{av}}$ & all Balmer \\
\hline $320 \pm 30$ & $390 \pm 10$ & $\sim 300$ & $360 \pm 20$ & $540 \pm 30$ \\
$410 \pm 50$ & $420 \pm 30$ & $310 \pm 40$ & $380 \pm 40$ & $580 \pm 50$ \\
$540 \pm 300$ & $430 \pm 100$ & $\sim 300$ & $490 \pm 200$ & $530_{-200}^{+300}$ \\
$\sim 300$ & $380 \pm 70$ & $\sim 300$ & $380 \pm 70$ & $470 \pm 40$ \\
$630 \pm 1200$ & $510 \pm 250$ & \pm 500 & $570 \pm 700$ & $530_{-160}^{+270}$ \\
$730 \pm 170$ & $440 \pm 90$ & $430 \pm 190$ & $530 \pm 150$ & $1100 \pm 300$ \\
\hline
\end{tabular}

the differential internal extinction, which is evaluated according to the galaxy morphological type (see RC3).

To explore the effect of the internal reddening, we additionally correct our optical and infrared magnitudes by $E(B-V)=0.06$ and $E(V-K)=0.16$. This reddening vector (see Fig. 5 ) is nearly parallel to the models, implying that the derived ages decrease, but without dramatically changing the metallicity of the best fitting model (see Sect. 4.2). We find that the photometric ages are $\sim 10 \%$ lower using $V-K$ and $14 \%$ lower using $B-V$, for the models with $Z=0.5 Z_{\odot}$. This variation represents an upper limit, as the actual internal reddening is likely to be lower than our conservative assumption. Hence we conclude that the optical-to-infrared colour distribution of our cluster sample mainly reflects the present stellar population.

\subsection{Stochastical fluctuations in the near-IR}

The bright infrared magnitudes of intermediate-age populations dominated by the TP-AGB phase are potentially affected by stochastic fluctuations due to the small duration of this phase $\left(t \sim 10^{6} \mathrm{yr}\right)$. This in turn implies small numbers of AGB stars. These effects are seen among the Magellanic Clouds clusters with a similar SWB parameter (i.e. a similar age) as a spread in the $V-K$ colours. The two-colour diagram of Fig. 5 already proves that large stochastical fluctuations are not present in our sample, which has a rather small scatter. This is reinforced by the close agreement between the ages determined using $B-V$ and $V-K$, shown in Fig. 7. The small fluctuations are a consequence of the high luminosity of most of our clusters, which implies a large sampled number of AGB members.
It is then interesting to estimate how many AGB stars are likely present in our clusters. Their number can be evaluated using the fuel consumption theorem.

In a stellar population of given age the number $N_{\mathrm{j}}$ of stars present in a certain evolutionary phase " $\mathrm{j}$ " is proportional to the total bolometric luminosity of the population $\left(L_{\mathrm{T}}\right)$ and to the duration of the phase $\left(t_{\mathrm{j}}\right)$, i.e.

$N_{\mathrm{j}}=B(t) L_{\mathrm{T}} t_{\mathrm{j}}$.

The coefficient of proportionality $B(t)$ is the specific evolutionary flux of the population (Renzini 1981; Renzini \& Fusi Pecci 1988; Maraston 1998), which is the number of stars entering or leaving any post-Main Sequence evolutionary stage per year and per solar luminosity of the population. Following Maraston (1998, see also Renzini 1999), the bolometric luminosity can be replaced by the total luminosity in any band $L_{\lambda}$, using the bolometric correction factor appropriate to the age and the metallicity of the population. The bolometric correction factors are by-products of population synthesis and are defined as $L_{\mathrm{T}} / L_{\lambda}$. Using the value $t \sim 10^{6}$ for the TPAGB lifetime and the photometric ages (for $Z=0.5 Z_{\odot}$ ) given in Table 4, we evaluate the number of TP-AGB stars for every cluster with a given $V$ and $K$ magnitude. The observed $V$ and $K$ magnitudes are converted to luminosities adopting a distance modulus of 34.04 (assuming $H_{0}=75 \mathrm{~km} \mathrm{~s}^{-1} \mathrm{Mpc}^{-1}$, following Schweizer \& Seitzer 1998) and a Galactic foreground extinction $A_{\mathrm{K}}=$ $0.36 * E(B-V)$ (see Sect. 2.2). The $V$ and $K$ magnitudes of the Sun are 4.83 and 3.41 respectively (Allen 1991). The expected numbers of TP-AGB stars are given in Table 6 . The TP-AGB phase in our clusters is well populated, the faintest clusters (W24 and and W19) having the smallest 


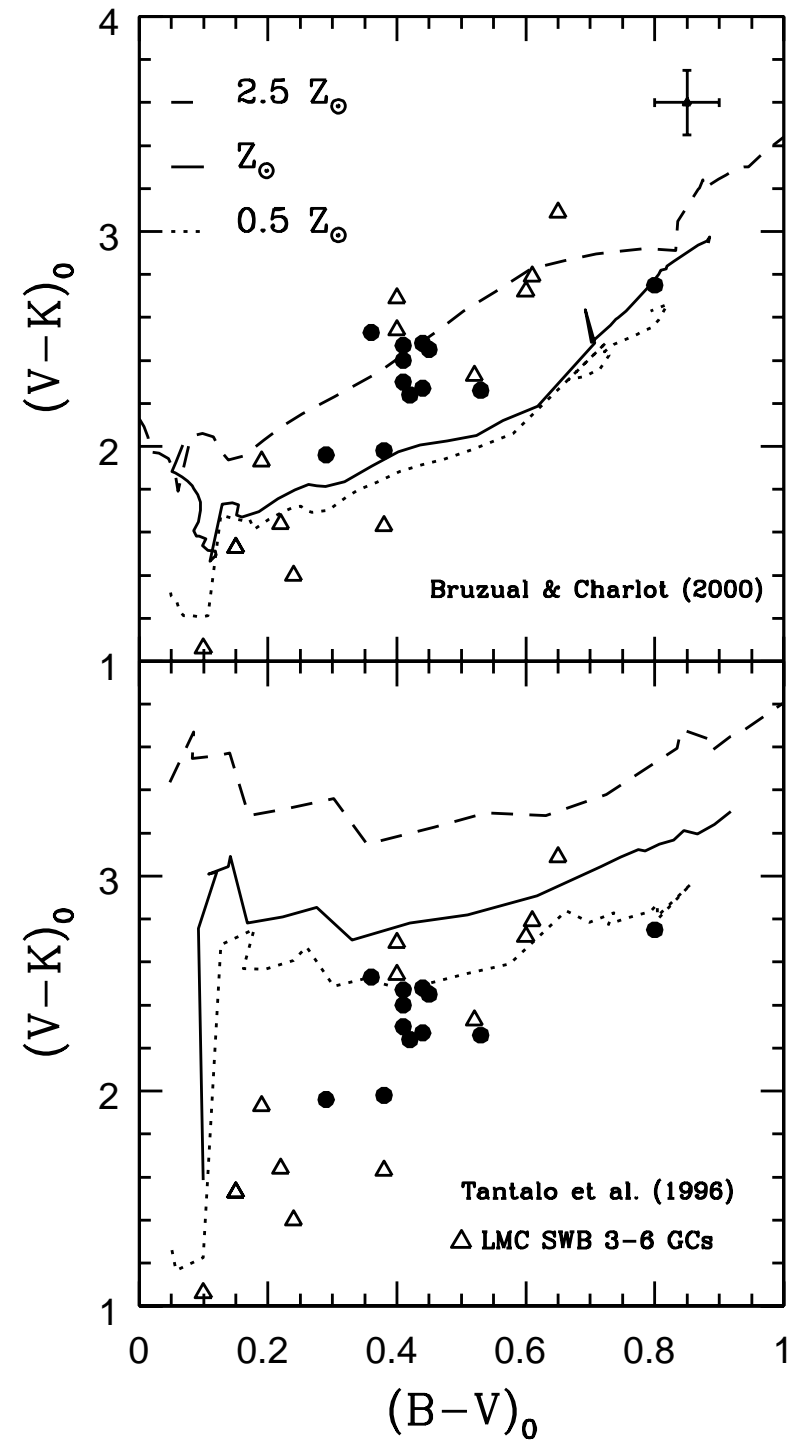

Fig. 6. The $V-K$ vs. $B-V$ diagram for the models of Bruzual \& Charlot (2000, private communication, upper panel) and Tantalo et al. (1996, lower panel). Data points are the same as in Fig. 5

numbers of TP-AGB stars. The expected stochastic fluctuations are given in the last two columns and range between $1 \%$ and $13 \%$. The corresponding magnitude fluctuations are of the same order, therefore smaller or comparable to the observational errors (see Table 1). A remarkable consistency is evident between the star counts obtained using the $V$ and the $K$ luminosities. This is not surprising because our SSPs are able to simultaneously reproduce the optical and IR colours very well, for $Z=0.5 Z_{\odot}$. Note that the expected numbers of stars are not significantly affected by changing the metallicity, because the specific evolutionary flux remains almost unchanged (see Renzini 1998; Maraston 2001, in preparation) and the main driver is the large luminosities of the clusters.

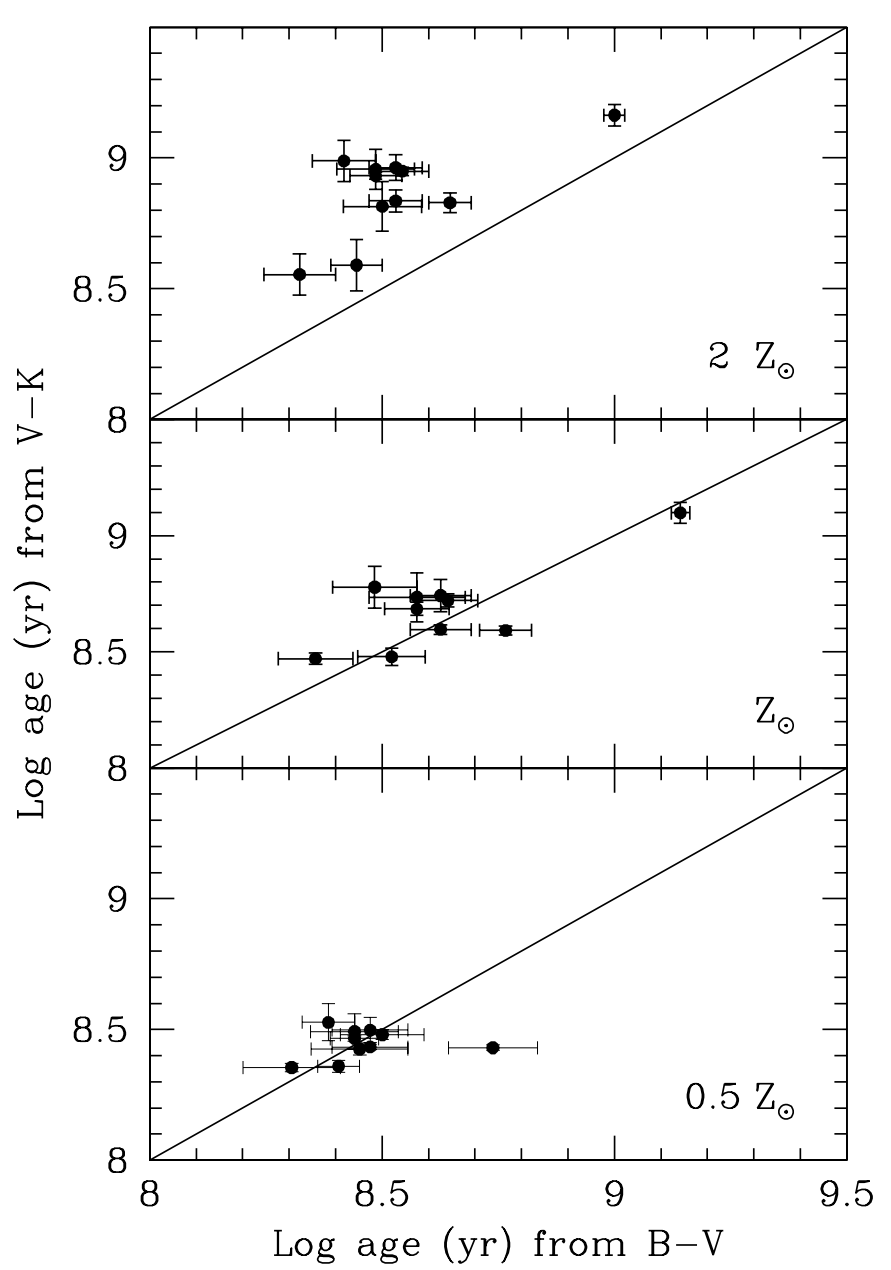

Fig. 7. Comparison between the ages derived from $B-V$ and $V-K$

\section{Metallicities and abundance ratios}

\subsection{The young star clusters}

The direct determination of cluster metallicities by measuring the strength of metallic lines (e.g. Mg, Fe, etc.) is complicated by two effects. The artificial broadening of the observed spectra, required in order to compare with the model ones, undesirably dilutes the metallic lines which are typically weak in the spectra of young stellar populations. This is aggravated by the low signal-to-noise of most of the spectra (see Table 3 and discussion in Schweizer \& Seitzer 1998). For these reasons we restrict the measurement of metallic lines to the best observed object, W3. We extend the exercise to objects W6 and W30, cautioning that these spectra are of much lower quality.

The equivalent widths of Mgb and Fe5270 are measured on the observed-broadened and model spectra. The comparison is made by measuring the indices on observed and model spectra exactly in the same way (see Sect. 2.5). For convenience, we adopt the definitions of line and continuum bandpasses given in Worthey et al. (1994). It is important to note that these model indices are not computed by means of the fitting functions given in Worthey et al. (1994). 


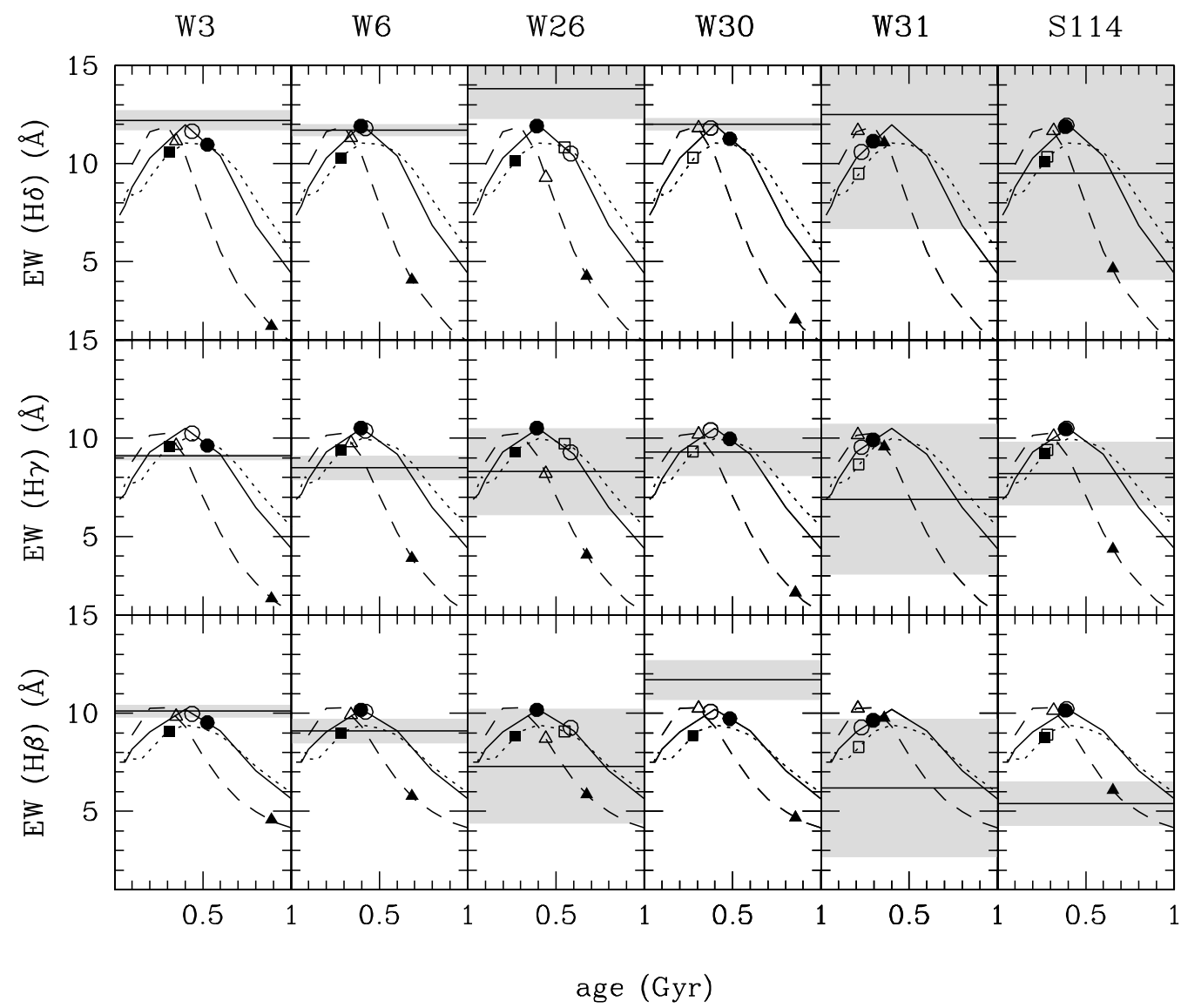

Fig. 8. Comparison between photometric and spectroscopic ages. $E W$ s measured from the observed spectra (named on the top of each panel) are represented as shaded areas, with widths of the same size as the errors of Table 3 . Curves are synthetic $E W \mathrm{~s}$ measured from a set of model spectra for SSPs with metallicity $0.5 Z_{\odot}$ (dotted line), $Z_{\odot}$ (solid line) and $2 Z_{\odot}$ (dashed line). Symbols on each curve show the photometric ages derived on the same models from $B-V$ and $V-K$ (open and filled symbols, respectively, cf. Table 4). Squares are used for $0.5 Z_{\odot}$, circles for $Z_{\odot}$ and triangles for $2 Z_{\odot}$

Table 6. The expected numbers of TP-AGB stars for the clusters listed in Table 1 . The ages for each cluster are from Table 4 for the model with $Z=0.5 Z_{\odot}$. Also quoted are the corresponding fluctuations

\begin{tabular}{lcccc}
\hline Name & $N_{\mathrm{j}}(V)$ & $N_{\mathrm{j}}(K)$ & $1 / N_{\mathrm{j}}(V)^{1 / 2}$ & $1 / N_{\mathrm{j}}(K)^{1 / 2}$ \\
\hline W3 & 4900 & 4700 & 0.01 & 0.01 \\
$\mathrm{~W} 6$ & 950 & 841 & 0.03 & 0.03 \\
$\mathrm{~W} 19$ & 73 & 73 & 0.12 & 0.12 \\
$\mathrm{~W} 22$ & 153 & 153 & 0.08 & 0.08 \\
$\mathrm{~W} 24$ & 56 & 56 & 0.13 & 0.13 \\
$\mathrm{~W} 25$ & 70 & 72 & 0.12 & 0.12 \\
$\mathrm{~W} 26$ & 490 & 300 & 0.05 & 0.06 \\
$\mathrm{~W} 27$ & 100 & 100 & 0.10 & 0.10 \\
$\mathrm{~W} 30$ & 1150 & 1200 & 0.03 & 0.03 \\
$\mathrm{~W} 31$ & 290 & 315 & 0.06 & 0.06 \\
$\mathrm{~W} 32$ & 220 & 290 & 0.07 & 0.06 \\
S114 & 225 & 222 & 0.07 & 0.07 \\
\hline
\end{tabular}

Figure 9 shows the synthetic $E W$ s of Fe5270 (bottom panel) and Mgb (top panel) for SSPs with various metallicities, as functions of age. The measured values for objects W3, W6 and W30 (symbols) are plotted versus the photometric ages given in Table 4 (see below). The vertical error bars are derived from the flux calibrated spectra by scaling our measurements errors to our measured $\mathrm{H} \beta$ errors, which in turn are matched to the $\mathrm{H} \beta$ errors given in Schweizer \& Seitzer (1998) (see Table 3).

We now comment on the individual clusters.

An excellent agreement is found for object W3 between the measured $E W \mathrm{~s}(\mathrm{Fe} 5270 \sim 0.83 \AA, \mathrm{Mgb} \sim 0.43 \AA)$ and the SSP with $Z=0.5 Z_{\odot}$, using the photometric age derived from this same model ( $\sim 300 \mathrm{Myr})$. By contrast, the photometric ages inferred from the $Z_{\odot}$ and the $2 Z_{\odot}$ models correspond to $E W$ s larger than the observed ones for both lines. The consistency between the results from photometry and spectroscopy indicates that W3 is a $\sim 300$ Myr old, $0.5 Z_{\odot}$ metallicity object with high likelihood. Note that measuring the Mgb and Fe $E W$ s on the original, higher resolution spectrum gives Fe5270 $0.84 \AA$ and $\mathrm{Mgb} \sim 0.48 \AA$ respectively, consistent with the values measured on the broadened spectrum.

In the case of object W6 $($ Fe5270 $\sim 1.27 \AA$ and $\mathrm{Mgb} \sim 0.56 \AA$ ), the best agreement is found when the 


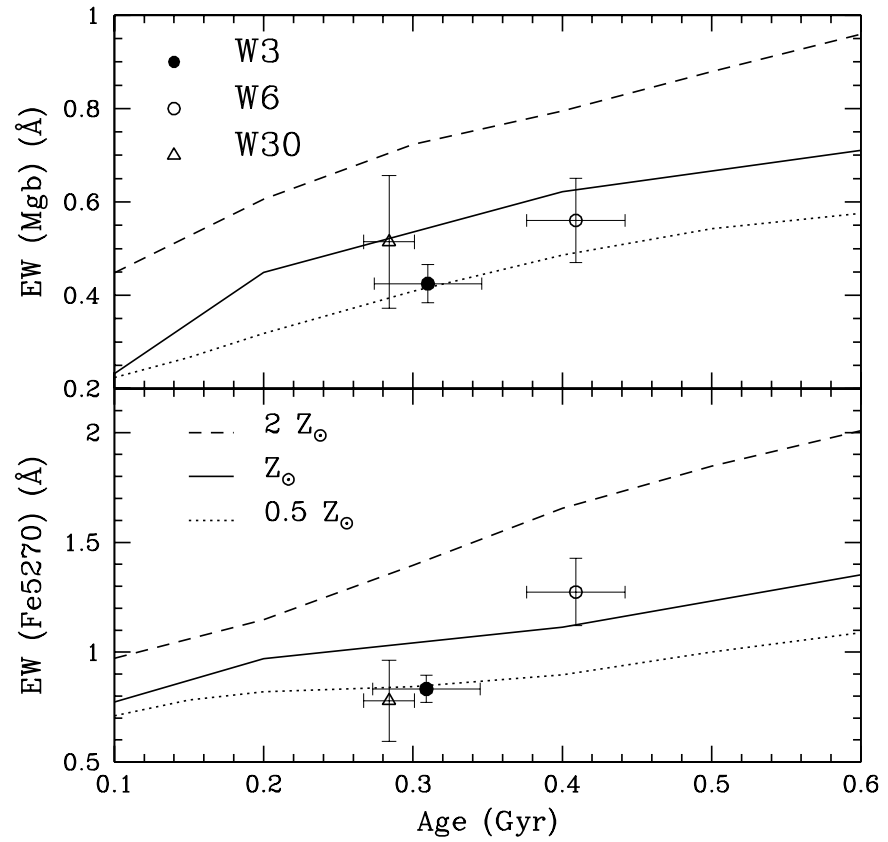

Fig. 9. Fe5270 and Mgb $E W \mathrm{~s}(\AA)$ for objects W3, W6 and W30, plotted vs. the photometric ages for $Z=0.5,1,0.5 Z_{\odot}$ respectively. Lines show the synthetic $E W$ s for SSPs with various metallicities

average photometric age derived from the $Z_{\odot}$ model is adopted. The other two metallicities agree less well.

Finally, we obtain Fe5270 0.78 $\AA$ and Mgb $\sim 0.51 \AA$ for object W30. These values do not allow us to clearly discriminate between half-solar and solar metallicity for this cluster (the former being slightly preferred), but metallicities much above solar appear to be ruled out.

Object W6 appears to be slightly more metal-rich than object W3. This is in qualitative agreement with the findings of Schweitzer \& Seitzer (1998), although they derive larger metallicities for both clusters.

This may be due to the use by Schweitzer \& Seitzer (1998) of model Lick indices based on the analytical fitting functions given in Worthey et al. (1994). As also noted by these authors, these functions are designed for old stellar populations, and it is not clear how well these can be applied to younger stellar populations.

The abundance ratios of $\alpha$-elements to iron-group elements carry information on the timescales over which star formation occurs, because $\alpha$-elements (e.g., $\mathrm{Mg}, \mathrm{O}$, etc.) are produced in type II supernovae (short-lived progenitors), while a significant fraction of iron comes from type Ia supernovae (long-lived progenitors). A solar $\mathrm{Mg} / \mathrm{Fe}$ implies star formation timescales of the order $10 \mathrm{Gyr}$, while $[\mathrm{Mg} / \mathrm{Fe}] \sim 0.2$ dex requires formation timescales $\sim 1$ Gyr (e.g., Thomas et al. 1999). If the observed Mg line-strength is stronger than predicted by SSP models based on solar abundance ratios, an enhancement of $\alpha$-elements is likely to exist, as is the case for luminous elliptical galaxies (see Sect. 6.3).

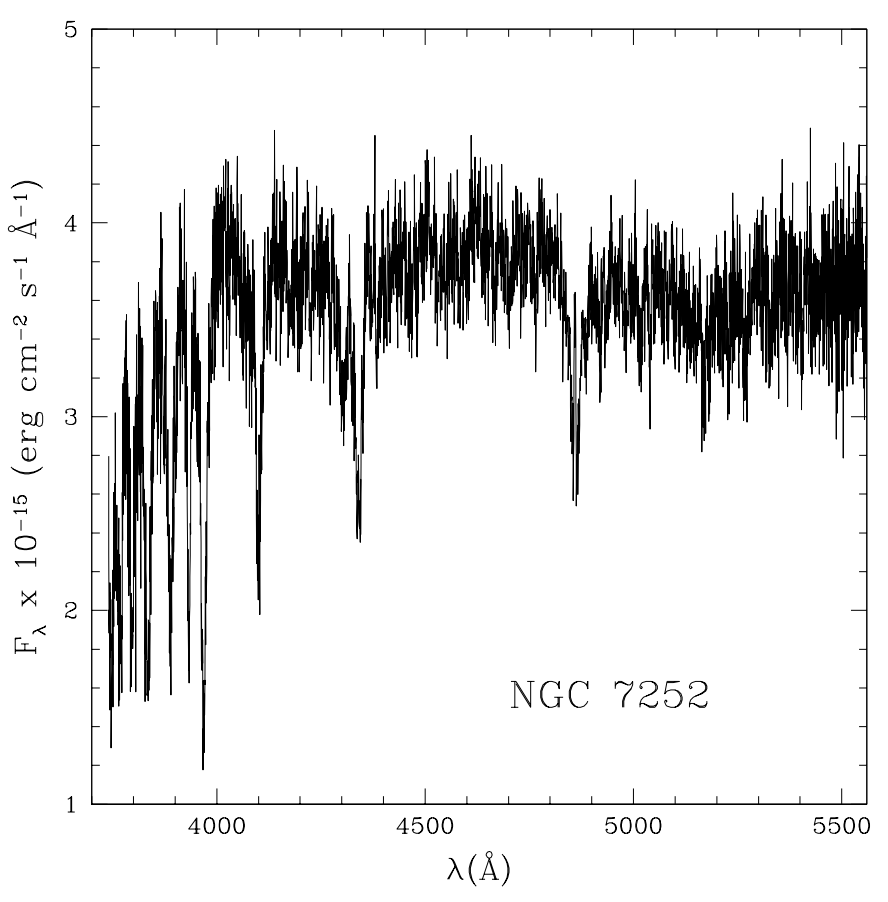

Fig. 10. Nuclear $\left(2^{\prime \prime} \times 2^{\prime \prime}\right)$ spectrum of NGC 7252 (F. Schweizer, private communication)

From Fig. 9 it appears that object W3 is not overabundant in $\alpha$-elements. This is what it is expected if the clusters form out of the highly Fe-enriched interstellar gas of the progenitor spirals (Thomas et al. 1999). This result is in agreement with the prediction by Fritze-v Alvensleben \& Gerhard (1994a), that any star clusters formed during mergers should have abundance ratios typical of the interstellar medium in the progenitor spirals.

\subsection{The diffuse light of NGC 7252}

If the young globular clusters form out of the interstellar medium associated with the merger event, it is interesting to investigate how the overall galaxy spectrum compares with the ones of the newly formed clusters. The spectrum of the diffuse light is produced by a mixture of stellar populations of various ages and metallicities, the light being dominated by the last episode of star formation. The interpretation by means of SSP models gives, by definition, only a measure of the average population mix. This analysis is nonetheless useful in order to identify the possible presence of features strikingly different from the cluster spectra. F. Schweizer kindly provided us with the spectrum of the central region of NGC 7252 (Fig. 10). The spectrum was taken in 1982 with the Las Campanas [Du Pont] 2.5-meter telescope, equipped with Shectman's Reticon. The aperture was $2^{\prime \prime} \times 2^{\prime \prime}$, centered on the nucleus, and the integration was $2000 \mathrm{~s}$, with a high dispersion $(1200 \mathrm{l} / \mathrm{mm})$ grating. This spectrum has been broadened (see Sect. 2.5) and the Balmer lines plus some metallic lines have been measured, using the same bandpass definitions as for the cluster spectra. For the 

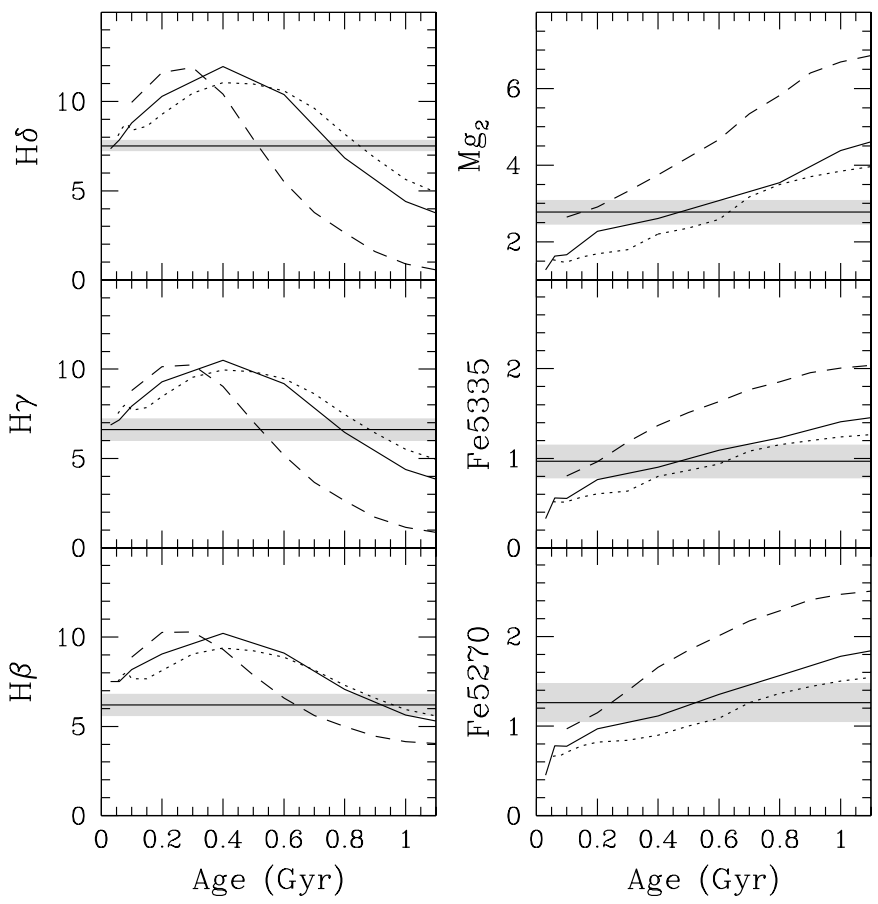

Fig. 11. Time evolution of Balmer line (left-hand plots) and metallic line (right-hand plots) $E W \mathrm{~s}(\AA)$ for SSPs with metallicities $0.5 Z_{\odot}$ (dotted line), $Z_{\odot}$ (solid line) and $2 Z_{\odot}$ (dashed line). The values measured on the spectrum of the central region of NGC 7252 (Fig. 10) are shown as straight lines

additional metallic lines Fe5335 and $\mathrm{Mg}_{2}$ used here, the index definitions given in Worthey et al. (1994) are adopted. In the case of the NGC 7252 nuclear spectrum, $\mathrm{Mg}_{2}$ is preferred to Mgb because the continuum of the latter can be increased by continuum hydrogen emission, common in young regions. This would produce an artificially deep Mgb line, because the continuum of the Mgb feature is defined to be adjacent to the line itself. $\mathrm{Mg}_{2}$ should suffer less from this effect because its continuum bandpasses are far enough from the absorption line.

The following values are obtained for the nuclear spectrum: $\mathrm{H} \delta \sim 7.52 \pm 0.3 \AA, \mathrm{H} \gamma \sim 6.62 \pm 0.6 \AA, \mathrm{H} \beta \sim 6.2 \pm$ $0.6 \AA, \mathrm{Mg}_{2} \sim 2.77 \pm 0.31 \AA, \mathrm{Fe} 5270 \sim 1.26 \pm 0.21 \AA$ and Fe5335 $0.96 \pm 0.19 \AA$.

In Fig. 11 these values (marked as straight lines) are compared to the predictions of SSPs with various metallicities, plotted as functions of age. Shaded areas represent errors.

The strengths of $\mathrm{H} \gamma$ and $\mathrm{H} \delta$ (left-hand panels) consistently suggest an age of: $\sim 870 \mathrm{Myr}$ for metallicity $0.5 Z_{\odot}$; $\sim 780 \mathrm{Myr}$ for solar metallicity; $t \sim 520 \mathrm{Myr}$ for metallicity $2 Z_{\odot}$. The slightly larger ages inferred from $\mathrm{H} \beta$ are because its value is lowered by filling from an emission line (clearly visible in Fig. 10). The strengths of the metallic lines (right-hand panels) also consistently constrain the age, which is, however, systematically lower than the one obtained from the Balmer lines, for every metallicity. The following value are found: $\sim 630 \mathrm{Myr}$ for metallicity
$0.5 Z_{\odot} ; \sim 480 \mathrm{Myr}$ for solar metallicity; $t \sim 240 \mathrm{Myr}$ for metallicity $2 Z_{\odot}$. The smallest discrepancy amounts to $28 \%$ for the $0.5 Z_{\odot}$ model; within the error bars the ages from Balmer lines and from metallic lines are consistent for the $0.5 Z_{\odot}$ model.

On average, the spectral features of the galaxy central diffuse light are consistent with a rather young average population with metallicity slightly sub-solar, in agreement with what is derived from the analysis of the star clusters. As in the case of the star clusters, the spectrum of the diffuse light does not show an enhancement in $\alpha$-elements. Simulations of the chemical evolution of composite stellar populations where a young component is born on top of an underlying old population during a spiral-spiral merger (Thomas et al. 1999) are in agreement with this result, in the sense that no $\alpha$-enhancement is observed in the resulting composite population.

Note that our results disfavour an IMF substantially flatter than Salpeter for the stars formed during the merger. A flat IMF would imply a larger number of SN II and fast enrichment of the gas in $\alpha$-elements. Therefore we would expect the newly formed stellar populations to be $\alpha$-enhanced (Thomas et al. 1999).

\section{Discussion}

\subsection{The recent star formation history of NGC 7252}

The derived ages and the age spread are compared with different models in order to constrain the nature of the progenitors and derive the star formation history during the merger event.

\subsubsection{The nature of the progenitors}

The ages derived for the observed young clusters confirm the small spread in age reported by Schweizer \& Seitzer (1998), see Fig. 12. Using different SSP models, we infer slightly lower cluster ages, ranging between 300 and 500 Myr. We confirm that cluster S114 for which Schweizer \& Seitzer could not conclude an age, does indeed fall in the same age range as the other clusters (300-400 Myr old, depending on the metallicity).

As previously pointed out by Schweizer \& Seitzer (1998), the ages are in reasonable agreement with predictions from $N$-body simulations for NGC 7252 for the star formation during a disk-disk merger (Mihos \& Hernquist 1996) which predict (for $H_{0}=75 \mathrm{~km} \mathrm{~s}^{-1} \mathrm{Mpc}^{-1}$ ) that the first pericenter contact happened $\sim 770 \mathrm{Myr}$ ago, the major burst of star formation occurring $\sim 100$ Myr years after this encounter, lasting $\sim 150$ Myr. We note that the predictions for star formation during the merger of bulge dominated disks differ from the predictions for a disk - disk encounter (Mihos \& Hernquist 1996). In bulge dominated mergers, galaxies appear stabilized against bar modes which tend to delay the gas inflow: star formation is predicted to happen $\sim 500 \mathrm{Myr}$ after the close 


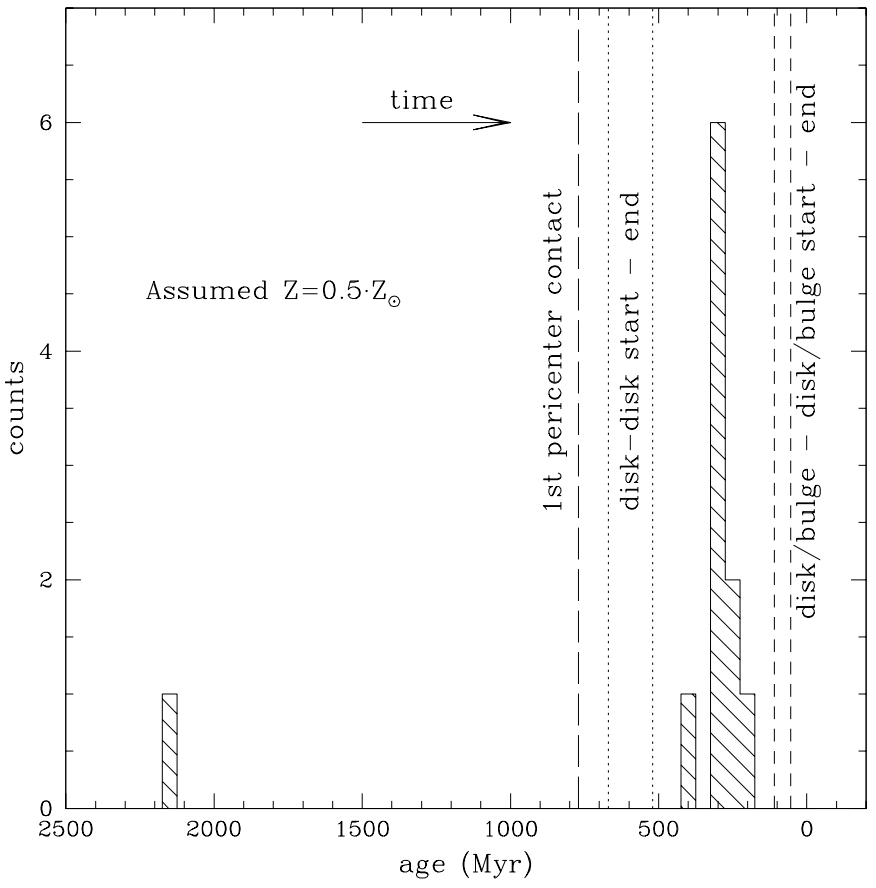

Fig. 12. Histogram of the mean cluster ages (as derived from photometry, assuming $Z=0.5 Z_{\odot}$ ). Time runs from left to right, the first pericenter contact $770 \mathrm{Myr}$ ago is marked as a dashed line. The predictions from the simulations of Mihos \& Hernquist (1996) are shown as dotted lines (marking the start and end of the main starburst in a disk-disk merger) and as short dashed lines (start and end of the main starburst in a disk/bulge-disk/bulge merger)

encounter and to be more violent but of shorter duration. In Fig. 12 (cf. Mihos \& Hernquist's Fig. 5) we show a histogram of the clusters ages (as derived from photometry for $Z=0.5 Z_{\odot}$ ) together with the predictions for a disk-disk merger and a bulge dominated merger. The beginning of the star formation does match either case: it starts slightly later as expected for a disk-disk merger, but slightly earlier than expected in a bulge dominated merger (although the timescale of the simulation can be rescaled by the distance to NGC 7252 and the mass of the progenitors). The duration of the star formation (taken as the dispersion of our ages $<1 \mathrm{Gyr}$ ) is around $50 \mathrm{Myr}$, closer to the prediction for a bulge dominated merger. With respect to the simulations, we cannot strongly conclude whether the progenitors of NGC 7252 were bulge dominated or not, but see evidence that they were not pure disks.

Fritze-von Alvensleben \& Gerhard (1994b) studied the integrated properties of the stellar light and reached the conclusion that NGC 7252 is best modelled by the merger of two massive Sc type spirals. Further, their study suggests that star formation took place 1.3 to 2 Gyr ago, well before the time of the first pericenter contact, namely 0.6-0.7 Gyr (Borne \& Richstone 1991). These conclusions are in agreement with our observations.

\subsubsection{Are there clusters in the 1 to 2 Gyr range?}

One cluster (W32) stands out in colour as older than the others. Unfortunately, no spectroscopy is available for it.

At face value, from the combination of its optical and near-infrared colours and our plotted SSPs, W32 appears to be a cluster with an age in the range 1 to 2 Gyr rather than being an old $(t \sim 10 \mathrm{Gyr})$ cluster belonging to the progenitors spirals. However, its optical colours $(B-V=0.80 \pm 0.04, V-I=1.08 \pm 0.04)$ could, according to SSP models, still be compatible with a metal-poor, old (12 to 15 Gyr) cluster. For example, Maraston (1998) models for $Z=0.006,15 \mathrm{Gyr}$, Gould et al. (1997) IMF predict $B-V=0.87, V-I=1.08$. In this case, the corresponding $V-K$ should be 2.59 , instead of $2.75 \pm 0.05$. Adding the near infrared colour therefore seems to exclude an old (10-15 Gyr) age. If W32 had an age of 15 Gyr its total luminosity, for a distance modulus of $(m-M)=34.04$ (assuming $H_{0}=75 \mathrm{~km} \mathrm{~s}^{-1} \mathrm{Mpc}-1$ ), would be $M_{V}=-12.5 \mathrm{mag}$ which is one to two magnitudes brighter than the brightest globular clusters in the Milky Way and M 31. There is no obvious reason for an abnormal $M / L_{V}$ which could translate the high luminosity into a normal mass (see Sect. 6.2). We conclude that W32 is likely to be a cluster in the age range 1 to 2 Gyr, and must have formed before the first pericenter contact of the two merging galaxies.

Finally, we checked whether W32 is an exception by verifying if our observed sub-sample is biased with respect to the total sample of Miller et al. (1997). A KolmogorovSmirnov test returns a probability of $\sim 90 \%$ that both samples are drawn from the same population. Miller et al.'s is assumed to be unbiased with respect to object colour in the range of interest, so that one could expect a few more clusters with similar ages to W32.

\subsection{The cluster masses}

Interestingly the young clusters, as already discussed by Schweizer \& Seitzer (1998), appear too bright by one to two magnitudes, compared to massive globular clusters, for the assumed distance. Could the distance to NGC 7252 have been overestimated? The systemic velocity of $4828 \pm 3 \mathrm{~km} \mathrm{~s}^{-1}$ for NGC 7252 was measured by Schweizer (1982). This value is in good agreement with the mean heliocentric velocity of $5083 \mathrm{~km} \mathrm{~s}^{-1}$ and velocity dispersion of about $430 \mathrm{~km} \mathrm{~s}^{-1}$ of the NGC $7284 / 85$ group, to which NGC 7252 belongs. Using the latest flow field model by the Key project (Mould et al. 2000), this translates into a distance around $68 \mathrm{Mpc}$ (assuming $\left.H_{0}=75 \mathrm{~km} \mathrm{~s}^{-1} \mathrm{Mpc}^{-1}\right)$, or $(m-M) \simeq 34.16$. It is therefore excluded that the distance to NGC 7252 has been significantly overestimated. Note that assuming $H_{0}=50 \mathrm{~km} \mathrm{~s}^{-1} \mathrm{Mpc}^{-1}$ would aggravate the problem.

Thus the young clusters in NGC 7252 are exceptionally luminous. The three brightest objects $\mathrm{W} 3, \mathrm{~W} 6$ and $\mathrm{W} 30$ have $M_{V}=-16.2,-14.4,-14.6$, respectively (for $H_{0}=$ $75 \mathrm{~km} \mathrm{~s}^{-1} \mathrm{Mpc}^{-1}$ and a distance modulus $m-M=34.04$; 
Schweizer \& Seitzer 1998). It is interesting to evaluate the cluster masses $M=(M / L) \times L$, adopting a model mass-tolight ratio. In order to compare with present day, $\sim 15 \mathrm{Gyr}$ old globular clusters, the fading of the young clusters due to aging has to be considered. We concentrate on object W3, which is the most luminous cluster.

Our synthetic stellar mass-to-light ratios take into account the contribution from stellar remnants, as described by Maraston (1998, see also Maraston 1999). The photometric age for object $\mathrm{W} 3$ is $t \sim 300 \mathrm{Myr}$ (for $Z=0.5 Z_{\odot}$, see Table 4). The corresponding mass-to-light ratio in $V$ for a Salpeter IMF is $\sim 0.28$, which translates in a present mass for $\mathrm{W} 3 \sim 7.210^{7} M_{\odot}$, a factor of $\sim 2.5$ lower than the value estimated by Schweizer \& Seitzer (1998) $\left(\begin{array}{lll}1.8 & 10^{8} & M_{\odot}\end{array}\right)$. The difference most likely results from Schweizer \& Seitzer (1998) assigning an age of $500 \mathrm{Myr}$ and a solar metallicity to W3. Both the older age and the larger metallicity increases the $M / L$ and hence the estimated mass. Part of the difference comes from the use of a model $M / L$ (Bruzual \& Charlot 1996 in Schweizer \& Seitzer 1998), in which stellar mass loss is not taken into account.

To explore the extent to which the mass can be lowered by changing the IMF, we additionally consider two cases. The first is a giant-dominated IMF with exponent -1.5 (in the notation in which Salpeter is -2.35). As already mentioned in Sect. 4.2 , the age for W3 changes to $280 \mathrm{Myr}$, corresponding to $M / L_{V} \sim 0.19$, hence a mass $4.910^{7} M_{\odot}$. This is lower by $\sim 32 \%$ than the one derived for the Salpeter case.

The second option is an IMF which is flattened at the low-mass end. We consider the one suggested by Gould et al. (1997), based on HST observations of disk $M$ dwarfs. The shape of this IMF is -0.9 for $M \leq 0.6 M_{\odot},-2.21$ for $0.6<M / M_{\odot} \leq 1$ and -2.35 for $m / M_{\odot} \geq 1$ (in the notation in which Salpeter is -2.35 ). The derived age for W3 is $\sim 300 \mathrm{Myr}$, as in the Salpeter case, because the effect on colours is negligible. The corresponding mass-tolight is $M / L_{V} \sim 0.155$, hence the present mass for W3 is $410^{7} M_{\odot}$. This is lower than the mass for the model with a Salpeter IMF by $45 \%$.

At an age of $15 \mathrm{Gyr}, \mathrm{W} 3$ will have a mass $\sim 6.310^{7} M_{\odot}$ for the Salpeter case $\left(M / L_{V} \sim 6.8\right), \sim 310^{7} M_{\odot}$ for the Gould et al. (1997) case $\left(M / L_{V} \sim 3.35\right)$ and $\sim 4.210^{7} M_{\odot}$ for the giant-dominated IMF $\left(M / L_{V} \sim 12.3\right)$. Note that the large mass-to-light ratios for the giant-dominated IMF are due to the larger number of massive stellar remnants (see Figs. 16, 17 in Maraston 1998). Taking also into account evaporation, the mass can be decreased by an additional 1\% (Binney \& Tremaine 1987).

Therefore the mass of the evolved W3 cluster will be at least $\sim 10$ times larger than the mass of $\omega$ Cen (2.9 $10^{6} M_{\odot}$, Harris 1996), which is the most massive cluster of the Milky Way. As already suggested by Schweizer \& Seitzer (1998), a dynamical estimate of the mass of W3, independent of population synthesis, is required to confirm these estimates. This is the subject of an ongoing project.

\subsection{Will NGC 7252 become a normal elliptical ?}

In the framework of hierarchical structure formation models (White \& Rees 1978; Kauffmann et al. 1993; Cole et al. 1994), elliptical galaxies are built up by merging disk systems. It is therefore interesting to know whether a merger-remnant like NGC 7252 will evolve into an object similar to a present day elliptical. A strong argument favouring this evolution is the observed light distribution in the inner part of NGC 7252, which closely resembles the $r^{1 / 4}$ de Vaucouleurs light profile of elliptical galaxies (Schweizer 1982). In spite of the fact that it is a recent merger, NGC 7252 already shows no sign of any major stellar disk component in its inner part. The colours of NGC 7252 will be close to the observed range of normal ellipticals after a time of 1 to 4 Gyr from the present, depending on when the star formation ceases (Fritze-von Alvensleben \& Gerhard 1994b). Yet there are two pieces of evidence against the evolution of NGC 7252 to a bona fide elliptical.

Elliptical galaxies have $\alpha$-enhanced average stellar populations (Worthey et al. 1992). This is found for ellipticals in dense environments (Mehlert et al. 2000; Kuntschner 2000) and in the field (González 1993; Trager et al. 2000; Beuing et al. 2000). Due to its very isolated position, NGC 7252 would become a field elliptical, likely an $L^{*}$ object. In Sect. 5 we showed that neither the cluster population nor the diffuse light of NGC 7252 show any sign of overabundance in $\alpha$-elements. Therefore, the present average stellar population of NGC 7252 cannot evolve into an average $\alpha$-enhanced stellar population, in contrast to the observations of present day ellipticals of the same luminosity. Note that abundance ratios provide a stronger constraint than colours because the latter can become sufficiently red if enough time for passive evolution is allowed after star formation stops. The abundance ratios of Fe and $\mathrm{Mg}$ instead are fixed when star formation stops and they cannot further change with passive evolution. Note that the convective mixing (dredge-up) during the red-giant phase does not alter the surface $[\mathrm{Mg} / \mathrm{Fe}]$ abundance ratio, because these elements are not synthetized in the interiors and therefore not dredged-up (see e.g. Renzini \& Voli 1981).

Another complication is the number of metal-rich globular clusters normalized to the galaxy light. It requires that a large number of new globular clusters have to form during the merger process (e.g. Forbes et al. 1997; KisslerPatig et al. 1999). We see from this work and from the work of others mentioned in the Introduction that new globulars indeed form during a merger-induced starburst. It is not, however, clear whether the number of new clusters is sufficient to be compatible with the number of red clusters observed in elliptical galaxies (see e.g. Harris 2000 for a review). As the efficiency in cluster production obviously increases with the gas content of the mergers (e.g. Kissler-Patig et al. 1998a), gas-rich mergers are better candidates. Gas-rich mergers, on the other hand do not produce $\alpha$-enhanced stellar populations, as discussed above. 
Furthermore the very young ages of the newly formed clusters (Sect. 4) imply a large age difference compared to the old cluster population belonging to the progenitor spirals. While a large number of studies have discovered bimodal GC colour and metallicity distributions of globular clusters in more than half of the early-type galaxies studied (Zepf \& Ashman 1993; Gebhardt \& KisslerPatig 1999), spectroscopic and photometric investigations indicate that both populations are old (Cohen et al. 1998; Kissler-Patig et al. 1998b; Kissler-Patig et al. 1998a; Kundu et al. 1999; Puzia et al. 1999). Ellipticals with GCs populations that are well-separated ( $>8 \mathrm{Gyr}$ ) in age seem to be rare. NGC 7252 will therefore remain an abnormal object for the present epoch even if, after the merger, it resembles an elliptical in several respects.

\section{Summary and conclusions}

Recent studies have shown evidence for a population of bright, young star clusters in the merger remnant NGC 7252 in which the AGB phase transition should be detectable. With this aim we obtained $K$ photometry for these clusters. This is a new approach, previous investigations have been confined to optical bands. Indeed, in intermediate-age populations $(0.2$ Gyr $\lesssim t \lesssim 1$ Gyr $)$ the AGB phase is the major contributor to the total energy. To analyze the data we used models for simple stellar populations in which the contribution of the AGB phase to the total light is calibrated on the intermediate-age globular clusters of the Magellanic Clouds.

- The $V-K$ vs. $B-V$ diagram predicted by our models describes the observed colours remarkably well and clearly shows the ongoing AGB phase transition among these clusters. This is the first detection of the AGB phase transition outside the Local Group;

- The photometric ages derived from $B-V$ and $V-K$ are in excellent agreement and are consistent with a metallicity $0.5-1 Z_{\odot}$ for the studied clusters;

- Most of the clusters appear to be 300-500 Myr old, consistent with previous age determinations based on optical spectroscopy;

- One cluster (object W32) stands out as being 1-2 Gyr old. If this object belongs to the same family of star clusters produced during the merger, then it must have formed before the first pericentral contact and cluster (and star) formation in a gas-rich disk-disk merger lasted at least 1-2 Gyr;

- We further analyze the spectral features of the best observed cluster W3 and of the diffuse light of the nucleus of NGC 7252, using spectroscopy from the literature (Schweizer \& Seitzer 1998; F. Schweizer, private communication). As expected from simulations of the chemical evolution of merging spirals (Thomas et al. 1999), neither stars nor clusters show an overabundance in $\alpha$-elements with respect to the iron group, in contrast to what is observed in elliptical galaxies of the same luminosity. This suggests that a present day disk-disk merger like NGC 7252 does not evolve into a present day elliptical. Our results disfavour an IMF substantially flatter than Salpeter during the merger-induced star formation, because this produces $\alpha$-enhanced stellar populations.

Acknowledgements. We are grateful to Bryan Miller for providing us with an electronic list of the UV and optical photometry, including unpublished data. Thanks also to François Schweizer for providing us with his spectra in electronic form. Santi Cassisi is acknowledged for the stellar tracks and isochrones used to compute the SSP models. We thank Daniel Thomas and Paola Marigo for helpful discussions. Finally, we are grateful to the anonymous referee for her/his relevant comments. Data presented herein were obtained at the W. M. Keck Observatory, which is operated as a scientific partnership among the California Institute of Technology, the University of California and the National Aeronautics and Space Administration. The Observatory was made possible by the generous financial support of the W. M. Keck Foundation. This work was supported in part by National Science Foundation grant number AST 9900732. CM is supported by the "Sonderforschungsbereich 375-95 für AstroTeilchenphysik" of the Deutsche Forschungsgemeinschaft.

\section{References}

Allen, C. W. 1991, Astrophysical Quantities (Athlone Press, London)

Bertin, E., \& Arnouts, S. 1996, A\&AS, 117, 393

Beuing, J., Mendes de Oliveira, C., \& Bender, R. 2000, MNRAS, submitted

Binney, J., \& Tremaine, S. 1987, in Galactic Dynamics (Princeton University Press)

Bono, G., Caputo, F., Cassisi, S., Castellani, V., \& Marconi, M. 1997, ApJ, 489, 822

Borne, K. D., \& Richstone, D. O. 1991, ApJ, 369, 111

Brodie, J. P., Schroder, L. L., Huchra, J. P., et al. 1998, AJ, 116, 691

Bruzual, G. A., \& Charlot, S. 1993, ApJ, 405, 538

Burstein, D., \& Heiles, C. 1984, ApJS, 54, 33

Cohen, J. G., Blakeslee, J. P., \& Ryzhov, A. 1998, ApJ, 496, 808

Cole, S., Aragon-Salamanca, A., Frenk, C. S., Navarro, J. F., \& Zepf, S. E. 1994, MNRAS, 271, 781

de Vaucouleurs, G., de Vaucouleurs, A., Corwin, H. G., et al. 1991, Third Reference Catalogue of Bright Galaxies (Springer Verlag), RC3

Fitzpatrick, E. L. 1999, PASP, 111, 63

Forbes, D. A., Brodie, J. P., \& Grillmair, C. J. 1997, AJ, 113 1652

Fritze-v Alvensleben, U., \& Gerhard, O. E. 1994a, A\&A, 285, 751

Fritze-v Alvensleben, U., \& Gerhard O. E. 1994b, A\&A, 285, 775

Frogel, J. A., Mould J. R., \& Blanco, V. M. 1990, ApJ, 352, 96

Gebhardt, K., \& Kissler-Patig, M. 1999, AJ, 118, 1526

Girardi, L., \& Bertelli, G. 1998, MNRAS, 300, 533

Gould, A., Bahcall, N. J., \& Flynn, C. 1997, ApJ, 482, 913

Harris, W. E. 1996, AJ, 112, 1487 
Harris, W. E. 2000, in Globular Cluster Systems, lectured for the 1998 Saas-Fee Advances School on Star Clusters (Springer Verlag)

Hibbard, J. E., \& Mihos, J. C. 1995, AJ, 110, 140

Holtzman, J. A., Faber, S. M., \& Shaya, E. J., 1992, AJ, 103, 691

Iben Jr., I., \& Renzini, A. 1983, ARA\&A, 21, 271

Lutz 1991, A\&A, 245, 31

Kauffmann, G., White, S. D. M., \& Guiderdoni, B. 1993, MNRAS, 264, 201

Kissler-Patig, M., Forbes, D. A., \& Minniti, D. 1998a, MNRAS, 298, 1123

Kissler-Patig, M., Brodie, J. P., Schroder, L. L., et al. 1998b, AJ, 115, 105

Kissler-Patig, M., Grillmair, C. J., Meylan, G., et al. 1999, AJ, 117, 1206

Kundu, A., Whitmore, B. C., Sparks, W. B., et al. 1999, ApJ, 513,733

Kuntschner, H. 2000, MNRAS, 315, 184

Lejeune, T., Cuisinier, F., \& Buser, R. 1998, A\&AS, 130, 65

Maraston, C. 1998, MNRAS, 300, 872

Maraston, C. 1999, in Star formation in early-type galaxies, ed. J. Cepa, \& P. Carral, ASP Conf. Ser., 163, 28

Mould, J. R., Huchra, J. P., Freedman, W. L., et al., 2000, ApJ, 529, 78

Marigo, P., in The chemical evolution of the Milky Way: stars versus clusters, 2000, ed. F. Matteucci, \& F. Giovannelli [astro-ph/9912341]

Matthews, K., \& Soifer, B. T. 1994, Infrared Astronomy with Arrays: the Next Generation, ed. I. McLean (Dordrecht: Kluwer Academic Publishers), 239

Mehlert, D., Saglia, R. P., Bender, R., \& Wegner, G. 2000, A\&AS, 141, 449

Mihos, J. C., \& Hernquist, L. 1996, ApJ, 464, 641
Miller, B. W., Whitmore, B. C., Schweizer, F., \& Fall, S. M. 1997, AJ, 114, 2381

Persson, S. E., Aaronson, M., Cohen, J. G., Frogel, J. A., \& Mathews, K. 1983, ApJ, 266, 105

Persson, S. E., Murphy, D. C., Krzeminzki, W., et al. 1998, AJ, 116, 2475

Puzia, T. H., Kissler-Patig, M., Brodie, J. P., \& Huchra, J. P. 1999, AJ, 118, 2734

Renzini, A. 1981, Ann. Phys. Fr., 6, 87

Renzini, A. 1998, AJ, 115, 2459

Renzini, A., \& Voli, M. 1981, A\&A, 94, 175

Renzini, A., \& Buzzoni, A. 1986 in Spectral Evolution of Galaxies, ed. C. Chiosi, \& A. Renzini (Dordecht, Reidel), 195

Renzini, A., \& Fusi Pecci, F. 1988, ARA\&A, 26, 199

Schweizer, F., ApJ, 252, 452

Schweizer, F. 1998, in Galaxies: Interactions and Induced Star Formation, ed. D. Friedli, L. Martinet, \& D. Pfenniger (Berlin: Springer), 105

Schweizer, F., \& Seitzer, P. 1998, AJ, 116, 2206

Searle, L., Wilkinson, A., \& Bagnuolo, W. G. 1980, ApJ, 239, 803

Thomas, D., Greggio, L., \& Bender, R. 1999, MNRAS, 302, 537

Trager, S. C., Faber, S. M., Worthey, G., \& González, J. 2000, AJ, in press [astro-ph 0005213]

van den Bergh, 1981, A\&AS, 46, 79

White, S. D. M., \& Rees, M. J. 1978, MNRAS, 183, 341

Whitmore, B. C., Schweizer, F., Leitherer, C., et al., 1993, AJ, 106, 1354

Worthey, G., Faber, S. M., \& González, J. 1992, ApJ, 398, 69

Worthey, G., Faber, S. M., González, J., \& Burstein, D. 1994, ApJS, 94, 687

Zepf, S. E., \& Ashman, K. E. 1993, MNRAS, 264, 611 